\title{
Radial Evolution of Sunward Strahl Electrons in the Inner Heliosphere
}

\author{
Allan R. Macneil ${ }^{1}$ - Mathew J. Owens ${ }^{1}$. \\ Mike Lockwood ${ }^{1}$. Štěpán Štverák ${ }^{2,3}$. \\ Christopher J. Owen ${ }^{4}$
}

Received: 5 July 2019 / Accepted: 30 December 2019 / Published online: 21 January 2020

(C) The Author(s) 2020

\begin{abstract}
The heliospheric magnetic field (HMF) exhibits local inversions, in which the field apparently "bends back" upon itself. Candidate mechanisms to produce these inversions include various configurations of upstream interchange reconnection; either in the heliosphere, or in the corona where the solar wind is formed. Explaining the source of these inversions, and how they evolve in time and space, is thus an important step towards explaining the origins of the solar wind. Inverted heliospheric magnetic field lines can be identified by the anomalous sunward (i.e. inward) streaming of the typically anti-sunward propagating, field-aligned (or anti-aligned), beam of electrons known as the "strahl". We test if the pitch angle distribution (PAD) properties of sunward-propagating strahl are different from those of outward strahl. We perform a statistical study of strahl observed by the Helios spacecraft, over heliocentric distances spanning $\approx 0.3-1$ AU. We find that sunward strahl PADs are broader and less intense than their outward directed counterparts; particularly at distances $0.3-0.75 \mathrm{AU}$. This is consistent with sunward strahl being subject to additional, path-length dependent, scattering in comparison to outward strahl. We conclude that the longer and more variable path from the Sun to the spacecraft, along inverted magnetic field, leads to this additional scattering. The results also suggest that the relative importance of scattering along this additional path length drops off with heliocentric distance. These results can be explained by a relatively simple, constant-rate, scattering process.
\end{abstract}

Keywords Magnetic fields, interplanetary $\cdot$ Magnetic reconnection, observational signatures $\cdot$ Solar wind

\footnotetext{
A.R. Macneil

a.r.macneil@ reading.ac.uk

1 Department of Meteorology, University of Reading, Reading, UK

2 Astronomical Institute CAS, Prague, Czech Republic

3 Institute of Atmospheric Physics CAS, Prague, Czech Republic

4 Mullard Space Science Laboratory, University College London, Dorking, Surrey, UK
} 


\section{Introduction}

It is instructive to split solar wind electron velocity distribution functions (VDFs) into three distinct components. These are the thermal "core", and the suprathermal "halo" and "strahl". The core and halo populations are quasi-isotropic. The strahl, however, takes the form of a field-aligned beam (Pierrard, Maksimovic, and Lemaire, 2001). Most commonly the strahl is aligned with the magnetic field in the anti-sunward ("outward") direction (Owens et al., 2017).

The strahl and halo inhabit a similar energy range of $\approx 70-2000 \mathrm{eV}$. As such, the strahl is typically observed as an enhancement with near parallel or anti-parallel pitch angles (PAs) over a more isotropic halo background. The fast solar wind is typically associated with strahl which is narrower and more pronounced than in the slow wind (Feldman et al., 1978; Pilipp et al., 1987a).

The strahl is most commonly thought to originate in the corona. Its formation can be explained by the evaporation of electrons in the high-energy tails of velocity distributions (e.g. Feldman et al., 1975; Pierrard, Maksimovic, and Lemaire, 1999) which can be formed through resonant wave-particle interactions (Vocks and Mann, 2003), although recently mechanisms such as instabilities have been explored (Che and Goldstein, 2014). The resulting hot solar wind electrons narrow into a beam as a result of adiabatic focussing, as they propagate outwards into a magnetic field which decreases in strength with heliocentric distance, $r$. Landi, Matteini, and Pantellini (2012) in fact show that strahl may also form locally in the solar wind, in simulations combining collisions and these expansion effects.

Studies which consider the heliocentric distance profiles of strahl properties have given insight into its evolution. Hammond et al. (1996), Graham et al. (2017), and Berčič et al. (2019) studied the pitch angle widths of the strahl beam, over distances of 1.3-3.8, 1-5.5, and $0.3-1 \mathrm{AU}$, respectively. Under purely adiabatic conditions, the strahl beam should continually narrow while travelling away from the Sun. However, these authors found that the width of the strahl beam at a range of energies predominantly tends to increase with heliocentric distance. Some process thus continually scatters the strahl in pitch angle, broadening the beam where it would otherwise narrow. Maksimovic et al. (2005) and Štverák et al. (2009), considering the solar wind at distances $0.3-1.5$ and $0.3-4$ AU respectively, both found that the density of the halo increases with radial distance at the expense of the strahl. These results too suggest that strahl electrons are scattered continually over the course of their propagation, but in this case into the halo. Other studies are indicative that suprathermal electrons are scattered during propagation. For example, Macneil, Owen, and Wicks (2017) observe that the energetic properties of suprathermal electrons observed at $1 \mathrm{AU}$ are far-removed from those predicted near the Sun, which is consistent with, e.g., scattering during propagation.

Owens, Crooker, and Schwadron (2008) created a simple model which encapsulates the competition between focussing and some scattering at a constant rate. They found that beyond $\approx 0.1 \mathrm{AU}$, strahl populations become broader the further they travel through the heliosphere because of the drop-off in degree of adiabatic focussing with distance and the cumulative effects of the constant scattering. Through considering these combined processes, the authors are able to produce a profile of strahl width against radial distance which agrees with that observed by, e.g., Hammond et al. (1996).

Much research on scattering mechanisms for the strahl has centred on wave-particle interactions. The most prominent example of this is resonant interaction with whistler-mode fluctuations (Vocks et al., 2005; Saito and Gary, 2007; Pagel et al., 2005; Viñas et al., 2010; Kajdič et al., 2016). Scattering through interactions with a range of other wave modes has 
also been investigated as additional potential scattering mechanisms (e.g. Pavan et al., 2013; Hellinger et al., 2014). More recently, the case for Coulomb collisions as a viable mechanism for strahl scattering has been made for lower energy $(\lesssim 200 \mathrm{eV})$ strahl electrons (Horaites, Boldyrev, and Medvedev, 2018).

The strahl is not universally observed to propagate in the anti-sunward direction. Socalled strahl "drop outs" have been observed by e.g., Pagel, Crooker, and Larson (2005), and theorised to occur as a result of the disconnection of the heliospheric magnetic field (HMF) from the Sun at both ends (McComas et al., 1989). Conversely, strahl may also propagate bidirectionally, in both the sunward (inward) and outward directions at once (Pilipp et al., 1987b). This "counterstreaming" can occur along closed loops in the HMF; where both ends may supply strahl electrons (Gosling et al., 1987). Finally, strahl may be observed to propagate in only the sunward direction. This can occur in instances where the HMF has become locally inverted or "kinked" relative to its dominant polarity (Kahler and Lin, 1994). Using these observational features, the direction of the strahl beam has been used to identify locally inverted, closed, and disconnected heliospheric flux through effectively remote-sensing the connectivity of the HMF (e.g. Gosling et al., 1987; Phillips et al., 1992; Crooker, Gosling, and Kahler, 2002; Pagel, Crooker, and Larson, 2005; Owens and Crooker, 2007; Crooker and Pagel, 2008).

\subsection{Inverted Heliospheric Flux and Solar Wind Origins}

In this study we are concerned primarily with instances of sunward directed strahl, due to its utility as an indicator of inverted HMF, a phenomenon which is important to understanding the origins of the solar wind. Interchange reconnection, reconnection between open flux and a closed loop, which results in the "opening" of the loop to the heliosphere, is often cited as an important mechanism in the production of the solar wind, particularly of the slow wind (Fisk, Schwadron, and Zurbuchen, 1998; Schwadron, Fisk, and Zurbuchen, 1999; Fisk, 2003; Antiochos et al., 2007; Crooker and Owens, 2012). It has been proposed that these newly-opened loops may be responsible for inverted HMF observed in the heliosphere. In particular, Owens, Crooker, and Lockwood (2013) suggest that reconnection with loops of height which exceeds the nominal height of solar wind release would produce a newly-opened magnetic field with a kink, which proceeds to propagate out into the solar wind (see their Figure 7). The kink gradually straightens at a rate controlled by the local Alfvén speed (Gosling et al., 2005). Alternatively, Owens et al. (2018) suggest that velocity shear along a magnetic field line resulting from the transfer of flux through interchange reconnection could drive a kink into the field. This kink would steepen as long as the velocity shear persists (see their Figure 4). In either case, the process of HMF inversion appears intrinsically tied to the production of the (slow) solar wind. Based on these suggestions, inverted HMF, as inferred by the presence of sunward strahl, has been used to infer the occurrence of interchange reconnection at the solar wind source in several studies (Crooker et al., 2004; Baker et al., 2009; Owens, Crooker, and Lockwood, 2013; Owens et al., 2018). In the future, as inner-heliosphere observations using Parker Solar Probe (Fox et al., 2016) and Solar Orbiter (Müller et al., 2013) are leveraged to discover the source of the slow solar wind, the utility of sunward strahl as an indicator for upstream reconnection will only grow. It is therefore timely to study in more depth the characteristics of inverted flux and sunward strahl, particularly in the inner heliosphere, to better understand their evolution and origins.

Sunward strahl would not be observed if Parker spiral theory applied at all times, giving HMF that is only in a "gardenhose orientation". Therefore, observations of sunward strahl can help identify which of many potential mechanisms are generating the "orthogardenhose" (i.e. $>45^{\circ}$ away from the Parker angle) field that is seen a sizeable fraction 
of the time (see review by Lockwood, Owens, and Macneil, 2019). In addition, inverted HMF generates "excess magnetic flux", whereby the unsigned heliospheric flux (the total of sunward and anti-sunward flux) does not fall off with an $r^{2}$ dependence (Owens et al., 2008; Lockwood, Owens, and Rouillard, 2009). Hence, observations of sunward strahl can be used to correct for this effect; allowing quantification of the true open solar flux entering the heliosphere (Owens et al., 2017). However, some inverted flux may remain undetected if the sunward strahl flowing along it is too strongly scattered; leading to an underestimation of the excess magnetic flux.

While above we discussed several studies into the radial trends of anti-sunward strahl, as yet no equivalent studies have been carried out for instances of sunward strahl. In this article, we employ suprathermal electron observations from the Helios spacecraft to identify, characterise, and compare statistically, anti-sunward and sunward strahl distributions measured at a range of heliocentric distances of $0.3-1$ AU. To our knowledge, this is the first such study to be carried out. In Section 2 we describe our analysis of the data and methods for identifying and characterising electron VDFs. In Section 3 we present our results comparing strahl widths and amplitudes, and halo backgrounds, between both sunward and outward strahl in fast and slow solar wind at three heliocentric distances. In Section 4 we discuss the implications of these results for the evolution of sunward strahl and inverted HMF, before drawing final conclusions in Section 5. We include two appendices, which supplement our interpretation of these results given some important limitations of the Helios data set.

\section{Data and Methodology}

The Helios spacecraft Plasma Experiment (E1) and Magnetic Field Experiment (E2) observed properties of in-ecliptic solar wind plasma and fields respectively. These measurements were made over heliocentric distances of 0.3-1 AU during the years 1974-1985 (Helios 1) and 1976-1979 (Helios 2). The two spacecraft measured a 2-dimensional slice of the electron velocity distribution with near-identical electron analysers (I2) (Schwenn, Rosenbauer, and Miggenrieder, 1975; Rosenbauer et al., 1977; Pilipp et al., 1987a). The analysers measured at 16 logarithmically-spaced energy bins, and eight evenly-spaced lookdirection bins in the ecliptic plane. A full scan was returned every $40 \mathrm{~s}$, but taking $16 \mathrm{~s}$ to build up. The Helios 1 angular coverage is illustrated in Figure 1a, for bins numbered $0-7$. In elevation, the response of the effective aperture for each bin was approximately Gaussian, with half width at half maximum (HWHM) of $9.5^{\circ}$ about the ecliptic plane. The azimuthal response of each bin was approximately uniform, with a width of $\pm 15^{\circ}\left( \pm 6.5^{\circ}\right)$ for Helios 1 (2) (Pilipp et al., 1987a). In this study we restrict observations to only Helios 1, which had a greater bin width and thus better angular coverage than Helios 2. After 1981, the availability of magnetic field measurements is very sparse, and so identifying the strahl is mostly impossible. We thus consider only the period $1974-1981$.

The Helios data are provided in the reference frame of the spacecraft, pre-corrected for spacecraft potential effects, which are in any case negligible for strahl electrons, which have energies far exceeding spacecraft potential values. Following Hammond et al. (1996) and Anderson et al. (2012), we analyse the strahl in the frame of the bulk solar wind. We shift the data into the bulk frame using radial and azimuthal velocities as obtained from the fitted Helios proton data set (Stansby et al., 2018). We then interpolate the data onto a grid in look direction and energy which is identical to the grid on which measurements were made in the spacecraft frame. Pitch angle distributions over single-energy slices can then be computed. Such an interpolation technique was applied in the production of pitch angle distributions in 

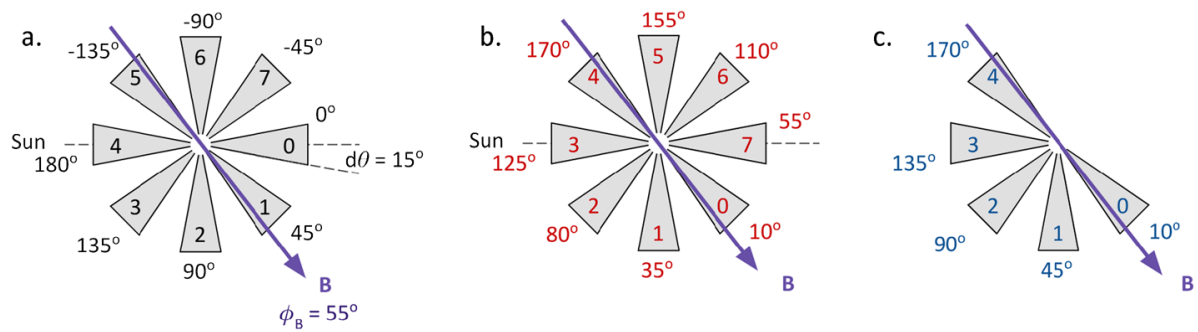

Figure 1 Schematics of Helios 1 electron detector bins in the ecliptic plane by look direction (a), pitch angle (b), and folded pitch angle (c). a.: Look-direction angular bins, with the central angle and bin number labelled. An in-ecliptic magnetic field vector is overlaid at an angle $\phi_{\mathrm{B}}$ in purple. The bin half width $d \theta$ is labelled. b.: Pitch angle bins defined and numbered as described in the text, given the magnetic field angle $\phi_{\mathrm{B}} . \mathbf{c} .:$ Folded pitch angle bins defined and numbered as described in the text.

the bulk solar wind frame for the Wind-3DP instrument. This method implicitly assumes that the electron VDFs are smoothly varying between energy and angle bins. In Appendix A, we demonstrate the importance of this correction for both the classification and characterisation of strahl.

The limited angular coverage of $I 2$ is problematic when identifying and characterising the strahl. For a HMF vector which does not fall sufficiently close to the centre of an angular bin, a very narrow strahl population will not produce any signal. To account for this, we follow Maksimovic et al. (2005) and Štverák et al. (2009) in removing data points from the study based on the direction of the magnetic field (i.e., we treat such data as a data gap). We remove all data where $\left|B_{\mathrm{z}} / B\right|>0.156$ (approximately corresponding to an elevation angle $\left|\theta_{\mathrm{B}}\right|>9.5^{\circ}$ out of the ecliptic). We also remove distributions where $\Delta \phi_{\mathrm{B}}$, the angle between the azimuthal component of the field, $\phi_{\mathrm{B}}$, and the central angle of the nearest angular bin, is $>d \theta=15^{\circ}$. Following this restriction of the data, a total of 178739 distributions remain. Although we restrict the data in this way, the narrowest strahl width which can be resolved with Helios $I 2$ is $\approx 15^{\circ}$ (see Appendix B) and so any inferences about strahl populations which are narrower than this are speculative.

Figure 2 shows a histogram of Helios 1 distributions split by heliocentric distance. This coverage results from a combination of factors including weighting from spacecraft orbit, gaps due to telemetry constraints (e.g., from the spacecraft passing behind the Sun, Roberts and King, 2008), and operational considerations. We expect only a few percent of the overall samples to be of sunward strahl (Owens, Crooker, and Lockwood, 2013), and so split the data into just three radial bins (dashed lines on the figure).

In previous strahl studies, improved angular coverage has been obtained by combining consecutive observations (e.g. Graham et al., 2017; Berčič et al., 2019). We do not take this approach, primarily because we find that many instances of sunward strahl are highly isolated in time, likely due to the strict criteria for inclusion above, data gaps, and occurrence rate of sunward strahl itself. It is thus not feasible to compute consecutive averaged distributions for the majority of the sunward strahl samples.

Throughout this study, unless otherwise stated, we specifically analyse and present results for suprathermal electrons measured in a representative strahl bin at energy $\approx 220 \mathrm{eV}$. We find that strahl electrons measured in bins of energy $\approx 150-600 \mathrm{eV}$ produce largely comparable results to this bin. This is supported by analysis of this same data set by (Berčič et al., 2019), who found that the width of strahl in this range did not show a strong energy dependence. We consider this limitation acceptable in order to streamline the analysis and presentation of the results of this study. 
Figure 2 Heliocentric distance histogram of electron distributions measured which meet the $\Delta \phi$ and $B_{\mathrm{Z}} /|B|$ criteria described in the text. The edges of the three distance bins used in this study are shown as vertical dashed lines.

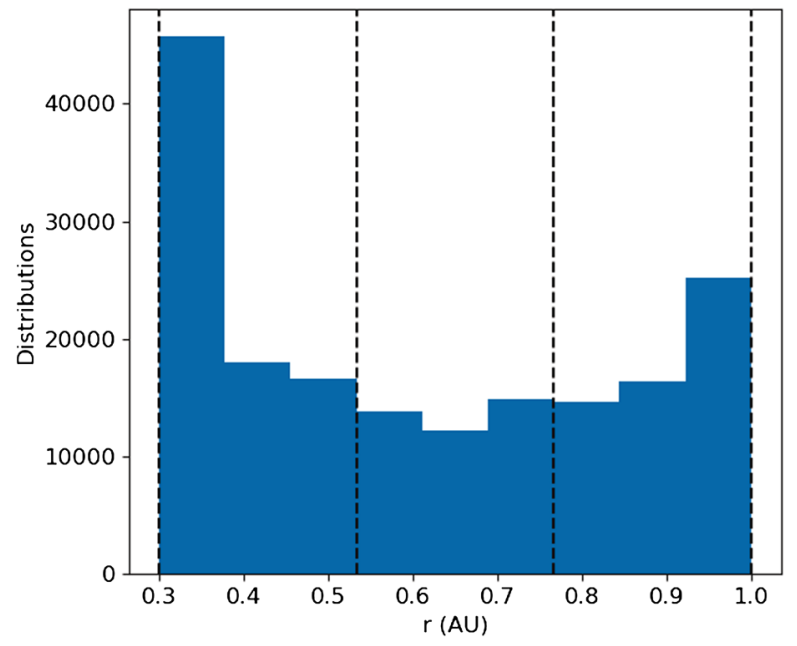

\subsection{Strahl Classification}

To classify the strahl, we first convert the 2-dimensional velocity distributions as a function of eight look directions (Figure 1a) to a function of eight pitch angles, as illustrated in Figure $1 \mathrm{~b}$. We then reduce the distributions from eight to five PA bins, by taking the mean of the pairs of PA bins which are closest to $45^{\circ}$ ( 1 and 7$), 90^{\circ}\left(2\right.$ and 6 ), and $135^{\circ}$ ( 3 and 5 ). This procedure was originally applied for the purposes of averaging Helios electron pitch angle distributions (PADs) by Maksimovic et al. (2005). We shall refer to this new PA distribution as the "folded" pitch angle distribution, or fPAD. A schematic of fPAD bins are shown in Figure 1c. fPAD bin PAs are $0-15^{\circ}$ for bin 0 and $165-180^{\circ}$ for bin 4 , depending on $\phi_{\mathrm{B}}$. The bin PAs are $45^{\circ}, 90^{\circ}$, and $135^{\circ}$ for bins 1,2 , and 3, respectively. Each fPAD now has three PAs in common, and so any algorithmic approach to classify the strahl is more consistent between different distributions than would be the case using full PADs.

The polarity of the HMF, in combination with the alignment of the strahl beam, is necessary to identify sunward strahl. In this study we define the polarity of the HMF, $B_{p}$, relative to the nominal Parker spiral angle, $\phi_{P}^{\text {nom }}$, calculated using the solar wind bulk velocity as in Heidrich-Meisner et al. (2016). We define anti-sunward $\operatorname{HMF}\left(B_{p}=1\right)$ as that for which $\phi_{\mathrm{B}}$ is within $\pm 90^{\circ}$ of $\phi_{P}^{\text {nom }}$, while all other angles correspond to sunward HMF $\left(B_{p}=-1\right)$. In previous studies which have identified inverted HMF, the polarity of the field has been defined as the sign of its radial component, $B_{r}$. However, in such an approach the size of a magnetic field deflection from $\phi_{P}^{\text {nom }}$ which constitutes an inversion is not constant with heliocentric distance, and so is not ideal for studies which examine radial trends.

We algorithmically classify each strahl distribution as either outward (anti-sunward), sunward (inward, indicating inverted flux), flat (isotropic at this energy; no strahl), or double (counterstreaming). Example PAD and fPAD cuts at $\approx 220 \mathrm{eV}$ for these strahl types are shown for reference in Figure 3. We make this classification for each fPAD at $\approx 220 \mathrm{eV}$. We label the phase space density value for each fPAD bin 0 to 4 as $f_{r 0}$ to $f_{r 4}$. We classify the fPADs by adapting the criteria developed by Crooker and Pagel (2008) for classifying strahl PADs measured by the Advanced Composition Explorer (ACE) at L1. For each PPAD, we compute two metrics based on their criteria. The ratio of the sum of $\log f_{r}$ for bins $<90^{\circ}$ to those for bins $>90^{\circ}$ is defined as the metric $M_{\mathrm{r}}$. $M_{\mathrm{r}}$ captures the strength of a unidirectional beam relative to the background (how far from 1 it is). $M_{\mathrm{r}}$ also classifies a strahl 
Figure 3 Four example distributions, $f$, at bin energy $\approx 220 \mathrm{eV}$, as a function of pitch angle, plotted in both PAD (error bars) and fPAD (dots) forms defined in the text. The distributions are normalised by $r^{2}$. Error bars are derived from the counting error associated with each measurement. Orange: outward strahl, green: sunward strahl, red: bidirectional strahl, purple: flat (isotropic) distribution.

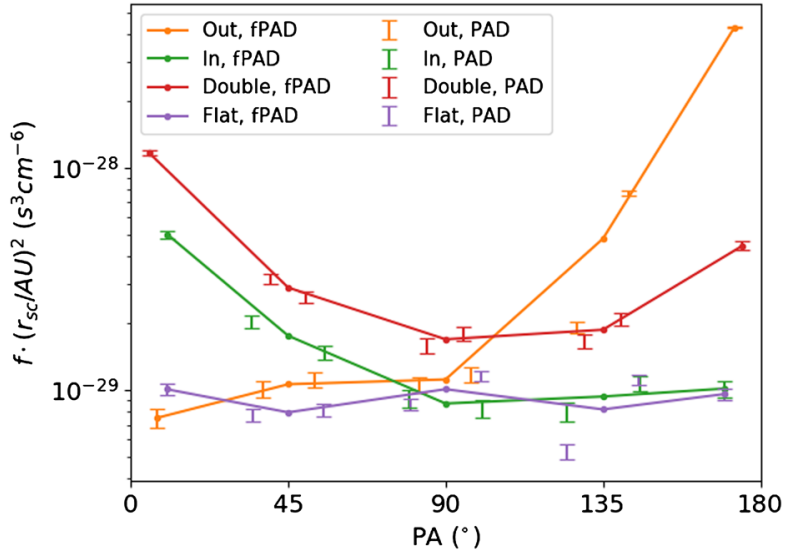

beam as anti-sunward or sunward when combined with $B_{p}$. For $B_{p}=1, M_{r}>1\left(M_{r}<1\right)$ indicates an anti-sunward (sunward) strahl, while for $B_{p}=-1, M_{r}>1\left(M_{r}<1\right)$ indicates a sunward (anti-sunward) strahl. We next define $M_{\mathrm{d}}$, the difference between the maximum and minimum of $\log f_{r}$. The fPAD is considered flatter as $M_{\mathrm{d}}$ approaches 0 . We also define two additional metrics. $M_{\perp / \|}$ is the ratio of the perpendicular bin, $\log f_{r 2}$, to whichever is greater out of $\log f_{r 0}$ and $\log f_{r 4} . M_{\perp / \|}>1$ when the perpendicular portion of the fPAD contains the largest flux, we exclude such periods from the classification. $M_{\mathrm{a}}$ is the ratio of $\log f_{r 2}$ to the smaller of the two possible strahl bins $\log f_{r 0}$ and $\log f_{r 4} . M_{\mathrm{a}}<1$ only when the electrons in the anti-strahl direction are stronger than the halo, the bidirectional case.

We classify the strahl using the above four metrics, $M$, based on the thresholds shown in the flowchart in Figure 4. As Crooker and Pagel (2008) pointed out, the choice of thresholds is effectively arbitrary as PAD properties are not split into well-defined populations, but they are in fact continuous between them. We define sensible thresholds by inspecting histograms of the metrics, and the PADs chosen following the classification process themselves.

Initial results of the above classification reveal a sub-population of PADs which are both flat, and feature significant enhancement in flux at bin energies $\gtrsim 150 \mathrm{eV}$. These distributions are removed from the study as they are clearly separated from each of the four valid strahl classes defined above. Determining the nature of this population is left to future work. We do note that enhanced suprathermal flux has been previously reported as an instrumental effect associated with use of the Helios 1 high gain antenna by Gurnett and Anderson (1977).

\subsection{Characterising Individual Strahl Distributions by Fitting}

In order to characterise different strahl populations, we carry out fits of PAD cuts at $\approx 220 \mathrm{eV}$ for all outward and sunward strahl distributions identified by the classification scheme. We account for the $r^{-2}$ drop-off of solar wind number density by normalising each distribution by $r^{2}$. We also flip the PADs with strahl centred on $180^{\circ}$, such that all PADs have the strahl centred around $0^{\circ}$. Next, following Hammond et al. (1996), we fit each PAD cut, $f_{E}$, with a Gaussian function, superposed over a constant background:

$$
f_{E}(\alpha)=A e^{-\alpha^{2} / 2 \sigma^{2}}+C,
$$

where $\alpha$ is the pitch angle, $\sigma$ is the width of the Gaussian, $A$ is its amplitude, and $C$ is a constant background. We further define the strahl width, $w$, as the half width at half maximum 


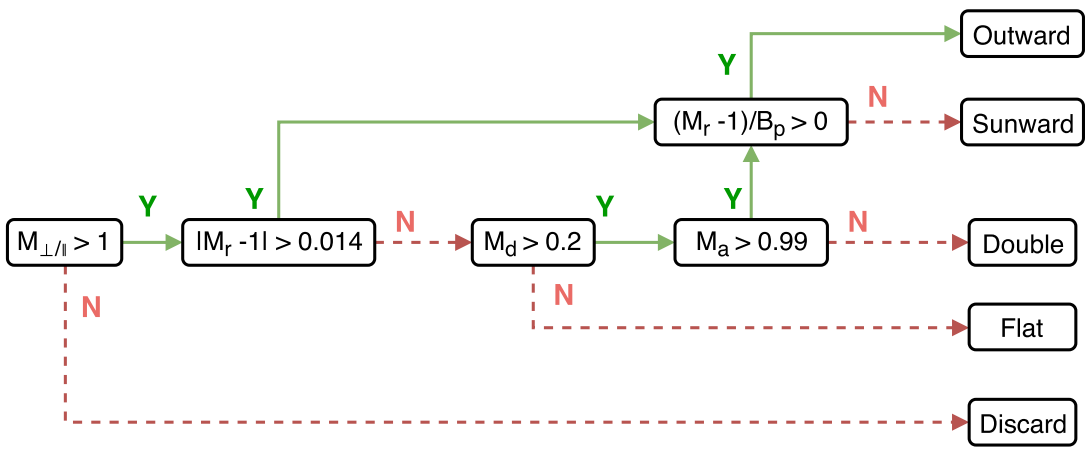

Figure 4 Flowchart showing the process for classification of strahl as outward, sunward, double, or flat, using the metrics described in the text and the polarity of the HMF, $B_{\mathrm{p}}$.

(HWHM) of the Gaussian:

$$
w=\sqrt{2 \ln 2} \sigma .
$$

The parameters $A$ and $w$ represent the strahl, while $C$ represents the halo as an isotropic background. This Gaussian fitting approach has been used previously by a number of authors studying strahl PA width (Hammond et al., 1996; Anderson et al., 2012; Graham et al., 2017), and it has been predicted analytically to be a suitable fit for strahl pitch angle distributions by Horaites, Boldyrev, and Medvedev (2018). We integrate the Gaussian portion of Equation 1 across all pitch angles to find the total area encapsulated by the Gaussian, $F_{s} \approx \sqrt{\pi / 2} \sigma A$. This value is indicative of the total strahl content at our chosen energy.

We carry out fittings using the non-linear least squares function curve_fit supplied with the SciPy library (Virtanen et al., 2019). Error estimates for the fitted parameters are derived from the square root of the diagonals of the covariance matrix, and we discard any fits where the error in $\sigma, A$, or $C$ exceeds $34 \%$ of the value itself. We use this high error tolerance to avoid discarding a large portion of data. We are thus able to compare probability distributions of the fitted parameters.

\section{Results}

\subsection{Strahl Classification}

To demonstrate the results of the strahl classification approach in Section 2.1, we plot all classified distributions for the year 1977 as fPADs at $220 \mathrm{eV}$ as a 2-dimensional histogram in Figure 5. Each fPAD is normalised such that the sum over all its bins is 1, and the distributions are transformed such that all outward strahl centre on $0^{\circ} \mathrm{PA}$, and all sunward strahl centre on $180^{\circ}$. As expected, the vast majority of strahl distributions are classified as outward, giving $>86 \%$ of all classified strahl observations. The number of distributions of sunward strahl and flat fPADs are comparable to one another, while there are around twice the number of double strahl distributions. The outward strahl fPADs shown in Figure 5 appear narrower and more intense than the sunward strahl fPADs, with most of the flux concentrated in the field-aligned bin. 

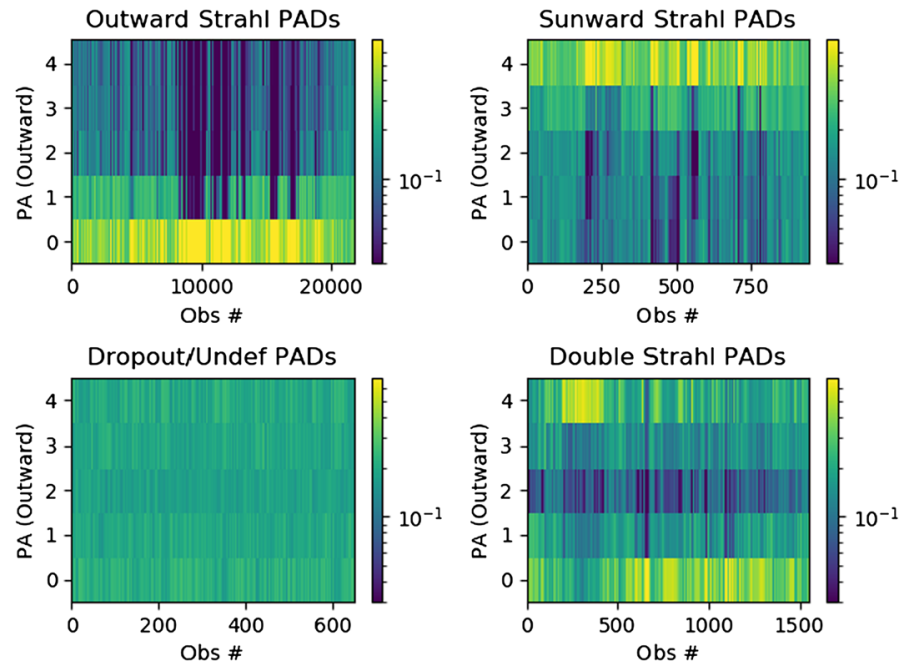

Figure 5 2-dimensional histograms of fPADs at $220 \mathrm{eV}$ by bin and distribution number during the year 1977, for strahl classified as outward, sunward (inward), dropout (flat), and double (bidirectional). $f$ is normalised such that all bins sum to 1. Distributions are flipped on the basis of the HMF polarity such that all outward strahl peak at bin 0 , and all sunward strahl peak at bin 4 .

Table 1 Table showing the number of distributions which are successfully fitted, separated by outward and sunward strahl, radial distance bin, and solar wind velocity bin.

\begin{tabular}{|c|c|c|c|c|c|c|}
\hline & \multicolumn{3}{|c|}{ Outward strahl } & \multicolumn{3}{|c|}{ Sunward strahl } \\
\hline & Fast & Slow & All & Fast & Slow & All \\
\hline $0.3-0.52 \mathrm{AU}$ & 12121 & 34745 & 46866 & 290 & 690 & 980 \\
\hline $0.52-0.76 \mathrm{AU}$ & 7176 & 15658 & 22834 & 393 & 447 & 840 \\
\hline $0.76-1 \mathrm{AU}$ & 11793 & 19380 & 31173 & 797 & 1100 & 1897 \\
\hline $0.3-1 \mathrm{AU}$ & 31090 & 69783 & 100873 & 1480 & 2237 & 3717 \\
\hline
\end{tabular}

\subsection{Fits of Individual Distributions}

Table 1 records the number of outward and inward/sunward distributions, which are fitted successfully based on the criteria in Section 2.2. These are split into bins of distance and solar wind velocity, where the split between "Fast" and "Slow" solar wind is $v_{\mathrm{sw}}=450 \mathrm{~km} \mathrm{~s}^{-1}$. We note that there are more distributions (both sunward and outward) measured under slow wind conditions than fast. This is consistent with the overall distribution of solar wind velocity for all available data points.

With increasing heliocentric distance, a greater fraction of distributions are classified as sunward. This result may be important for the evolution of inverted flux in the heliosphere. However, we do not comment on this further here, as our strict selection process may preferentially remove certain types of strahl. Further, as we do not know the origins of the anomalous flat distributions which we exclude from the study in Section 2.1, we are not as confident to comment on occurrence of distributions as we are to characterise properties. We intend to pursue this line of investigation as future work.

Figure 6 plots normalised histograms of the fitted strahl width, $w$, strahl amplitude, $\log (A)$, and background, $\log (C)$, for outward and sunward strahl at $220 \mathrm{eV}$, separated into the three distance bins. We display $A$ and $C$ logarithmically as the probability distributions 

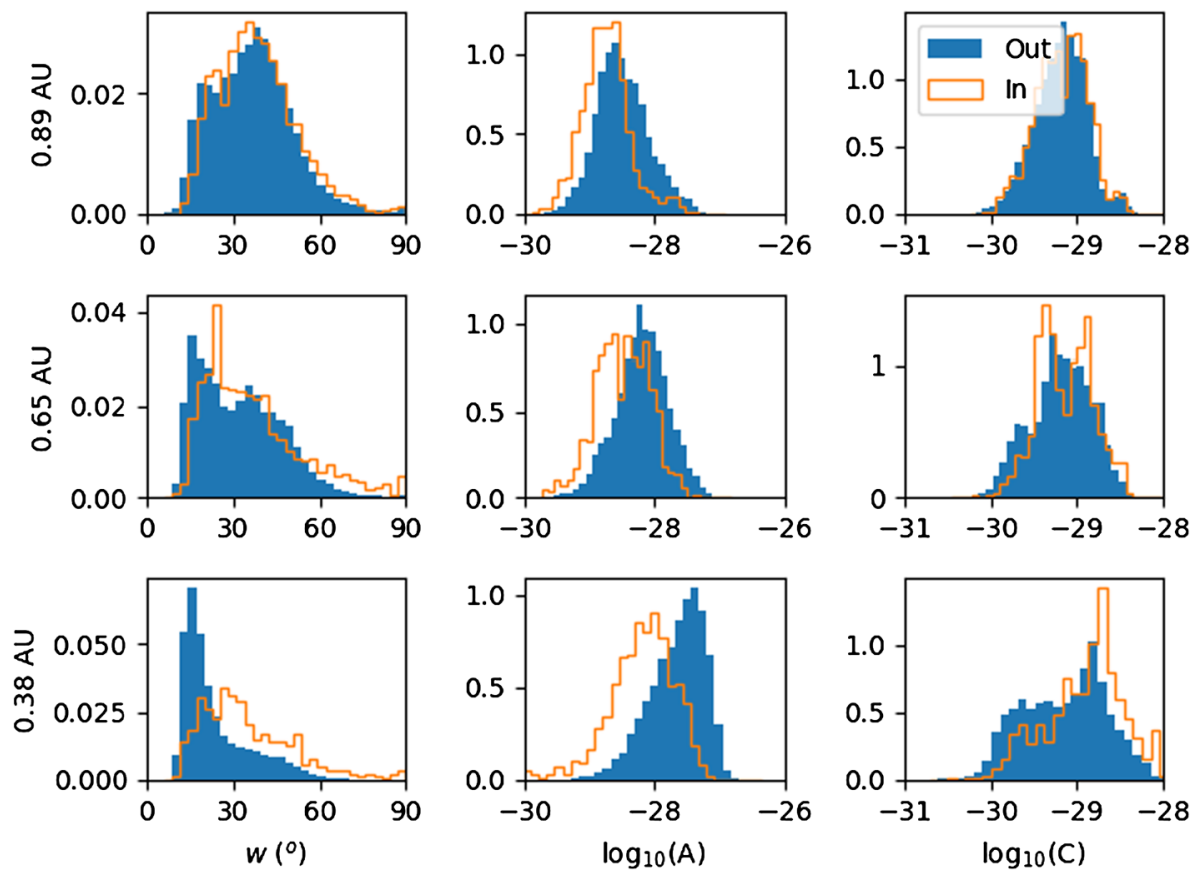

Figure 6 Normalised histograms of $w, \log (A)$, and $\log (C)$ for outward (blue) and sunward (orange) strahl data at three distance bins, for all wind speeds combined. The mean sampled distance of each bin is listed at the left of each row of panels.

of these values more closely resemble a log-normal distribution than a normal one. Here and in all subsequent figures, these parameters have been calculated using the distancenormalised distribution, $f r^{2}$, and so these results are comparable across distances, save for any propagation effects.

Examining the distributions of outward strahl widths, $w$, we immediately note a rapid drop-off towards lower values, at around $15^{\circ}$ in the inner two distance bins. This contrasts with the upper limits of $w$, which tail off gradually. Further, the distributions peak just above the $\approx 15^{\circ}$ cut-off. These features can be understood under our evaluation of the Helios 1 electron data set and fitting procedure in Appendix B, in which we consider the effects of the instrument angular bin width. We find there that distributions of underlying width $w \lesssim 15^{\circ}$ are probably rejected by the fitting procedure or, as indeed appears to be the case from Figure 6, fitted with overly-wide values of $w$. This leads to a depletion of widths $w<15^{\circ}$ and a "piling-up" at $w \approx 15^{\circ}$, as shown in the figure.

Aside from issues at $w \lesssim 15^{\circ}$, we note the large spread in all parameters which is present for both outward and sunward strahl. For the inner distance bins, sunward strahl is notably wider than outward. Sunward strahl also features consistently a lower amplitude, $A$, than outward strahl, and is associated with greater $C$ in the inner distance bin, which features a very strong and narrow peak.

To investigate sample size effects, we have also produced distributions of outward strahl parameters using randomly selected sub-samples of the outward PADs. For each bin, we take the same number of samples of outward strahl PADs as there are sunward strahl PADs, as indicated by Table 1 . We then compare the sub-sampled distributions to the full distributions shown in Figure 6, and find that each match closely. 

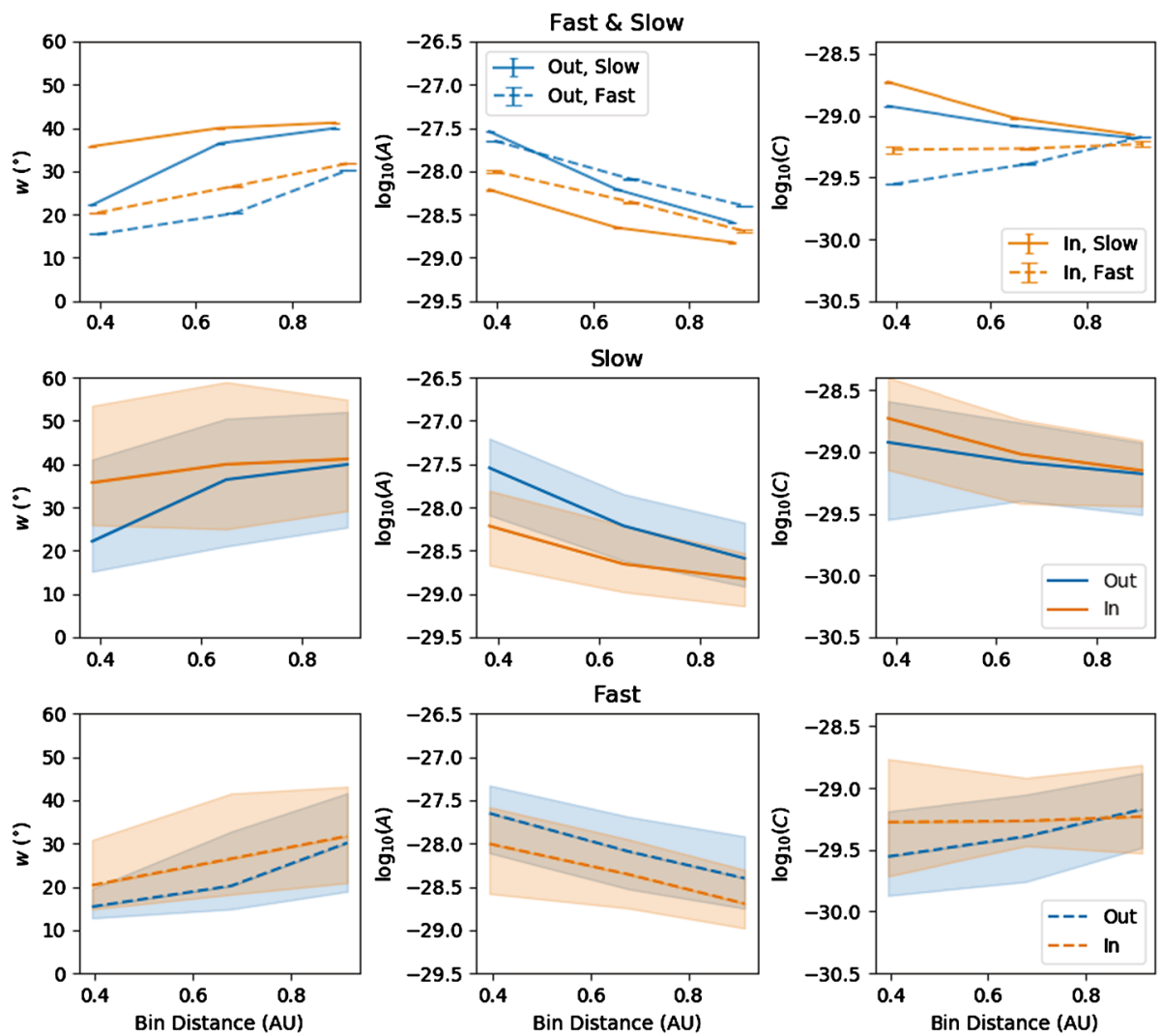

Figure 7 Plots of median $w, \log (A)$, and $\log (C)$, against heliocentric distance bin for slow and fast wind, for outward and sunward strahl. In the top row, error bars correspond to the standard error, and thus correspond to the sampling uncertainty. The lower two rows show only slow and fast wind, with percentiles of $15.9 \%$ and $84.1 \%$ derived from the underlying distributions shown as shaded regions, and thus correspond to the natural variability. Bin distances are the mean distance of all the measurements which are included in the respective distance and velocity bin.

We plot the median values of the above parameter distributions, now further split into fast and slow wind (cut-off at $v_{\mathrm{sw}}=450 \mathrm{~km} \mathrm{~s}^{-1}$ ), against distance in the top row of plots of Figure 7. The error bars correspond to standard errors in the median, and they are at times too small to be clearly visible. The middle and lower rows of Figure 7 show the same parameters, for only slow and fast wind respectively. Percentiles $\pm 34.1 \%$ at either side of the 50th percentile (the median), $84.1 \%$, and $15.9 \%$ are shown as shaded regions. In a Gaussian distribution, one standard deviation corresponds to $\pm 34.1 \%$ about the median, and so we use this percentile value as a rough equivalent, given the non-Gaussian distributions found here. The shaded areas thus capture the large spread which is present in the histograms of Figure 6, and overlap between sunward and outward populations. $A$ is the parameter with most clear separation between these two populations.

Figure 7 shows that, on average, outward strahl increases in width with heliocentric distance. This trend is present for fast and slow wind. The total increase in width is slightly greater for slow wind (median slow width increases by $\approx 18^{\circ}$, while fast width increases by $\approx 15^{\circ}$ ). For both sunward and outward strahl, the median width during fast solar wind is narrower than that during slow wind. Sunward strahl widths do not show such strong varia- 
Figure 8 Plots of median $\sigma A$, and $\log (A+C)$ against heliocentric distance bin for slow and fast wind (solid and dashed lines, respectively), and for outward and sunward strahl (blue and orange lines, respectively). The error bars correspond to the standard error in the median.
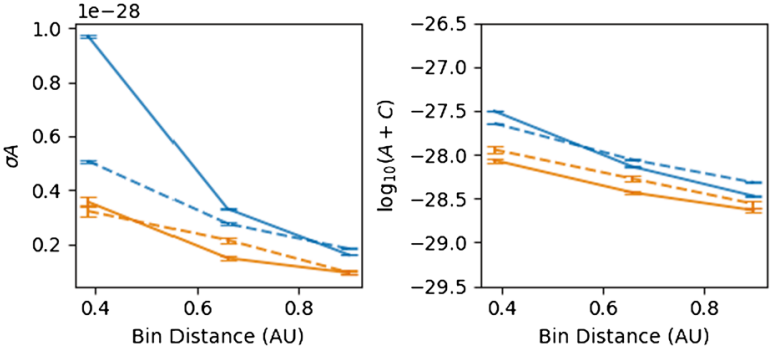

tion with solar wind speed or radial distance. Although an overall increase is still found for both wind types, the slow wind samples in particular vary very little with radial distance. For both fast and slow wind, sunward strahl is wider than outward strahl in the innermost distance bin, but tends to converge to outward values by the outermost distance bin.

Across all distances, outward strahl has greater median $A$ than sunward strahl. Both exhibit decreasing $A$ with heliocentric distance. The total decrease approaches an order of magnitude for slow wind outward strahl, and a factor of $\approx 5$ for all other types. Fast wind is associated with strahl of greater $A$ than slow, apart from in the innermost outward distance bin. $A$ for sunward and outward strahl also appears to weakly converge with radial distance, particularly comparing slow wind.

While $C$ associated with outward strahl increases with radial distance in the fast wind, it instead weakly decreases in slow. Meanwhile, $C$ associated with sunward strahl remains nearly constant in the fast wind, and again decreases in the slow. We also note that for both sunward and outward strahl, the associated values of $C$ tend to converge at $r \approx 1$ AU.

Figure 8 plots the medians of two parameters obtained from combinations of width, amplitude, and background, $\sigma A$ and $\log (A+C)$, against $r$ ( $\sigma$ is the standard deviation of the fitted Gaussian as in Equation 1). The error bars are the standard error in the medians for the distributions of $\sigma A$ and $\log (A+C)$. We plot $\sigma A$ as it is proportional to the total area below the Gaussian portion of Equation 1 (see Section 2.2). At all distances, $\sigma A$ is greater for outward strahl than sunward. We find that $\sigma A$ drops off for both with bin distance, but this drop is far sharper for outward strahl than sunward, and for slow wind than fast, between the $\approx 0.4$ and $0.6 \mathrm{AU}$ bins. Outward and sunward strahl lines for $\sigma A$ again tend to converge as a result of their differing trends. The parameter $\log (A+C)$ represents the evolution of the total amplitude of $f_{E}$ in Equation 1 (whereas $A$ is the strahl component only). We find that, as $C$ is far smaller than $A$, the plotted values and their trends are very similar to $\log (A)$.

\section{Discussion}

\subsection{Caveats}

Some caveats are necessary to inform our interpretation of the results of this study, and when discussing them in the context of other work. First, all results apply for strahl samples measured when the magnetic field is approximately in the ecliptic plane. This preferentially excludes populations which are present during periods of enhanced southern magnetic field, $B_{\mathrm{z}}$, e.g. sections of interplanetary coronal mass (ICME) flux ropes, stream interaction regions (SIRs; see, e.g., Lei et al., 2011), and periods of strong magnetic fluctuation. ICME flux ropes are ejecta and not part of the background solar wind, while SIRs and strong magnetic fluctuations that may be associated with a more scattered or otherwise distorted strahl. 
Table 2 Comparison of strahl widths derived from the present study and Berčič et al. (2019) at $220 \mathrm{eV}$, and Hammond et al. (1996) at $225 \mathrm{eV}$, split into fast and slow solar wind, and by the innermost and outermost distance bins of this study. Berčič et al. (2019) results are split by core parallel electron plasma beta, $\beta_{e c \|}$, instead of speed, and the quoted inner and outer results are for the range of widths spanned by the inner and outer two distance bins of their study. The Hammond et al. (1996) results are extrapolated to 0.91 AU from Ulysses observations as described in the text.

\begin{tabular}{lllll}
\hline & & This study & Berčič et al. & Hammond et al. \\
\hline Fast/Low $\beta_{e c \|}$ & $\approx 0.3-0.52 \mathrm{AU}$ & $15^{\circ}$ & $20-22.5^{\circ}$ & - \\
& $\approx 0.76-1.0 \mathrm{AU}$ & $30^{\circ}$ & $30-37.5^{\circ}$ & $27^{\circ}$ \\
Slow/High $\beta_{e c \|}$ & $\approx 0.3-0.52 \mathrm{AU}$ & $22^{\circ}$ & $30-42^{\circ}$ & - \\
& $\approx 0.76-1.0 \mathrm{AU}$ & $39^{\circ}$ & $45-52.5^{\circ}$ & - \\
\hline
\end{tabular}

The effective removal of these periods for this study leaves a subset of data which better represents the ambient, quiet, solar wind.

Second, as we show in Appendix B, the limited angular coverage of $I 2$ leads to the pileup, followed by rapid drop-off, of points in the distributions of $w$ at $\approx 15^{\circ}$. The "true" distribution of widths likely extend to narrower values below the drop-off. We stress again that our results only strictly apply to strahl of width $>15^{\circ}$. The presentation of median, as opposed to mean, parameters in Figure 7 mitigates the impact of this pile-up on our results, however.

Third, only a small number of total sunward strahl samples are available in this study (compared to outward strahl). However, random sub-sampling of outward strahl PADs in Section 3.2 demonstrates that this number of samples is sufficient to produce robust width results, and so the sunward strahl results are not an artefact of poor statistics.

Finally, we note that our analysis neglects cooling undergone by solar wind electrons during their outward propagation. Core electron temperature, $T_{c}$, decreases with heliocentric distance (e.g. Štverák, Trávníček, and Hellinger, 2015). The breakpoint energy, $E_{b}$, between the core and halo strahl has been predicted to be $\approx 7 k_{B} T_{c}$ by Scudder and Olbert (1979), and for the strahl in particular it is found observationally to be $\approx 2-4 k_{B} T_{c}$ between $0.3-$ 1 AU (Štverák et al., 2009). The approach in this study (and those of Hammond et al., 1996; Graham et al., 2017; Berčič et al., 2019) which compares cuts through electron VDFs in the same energy bin at each distance, neglects this cooling. As such, we do not necessarily observe equivalent cross-sections of the strahl in each distance bin. However, Berčič et al. (2019) observe that strahl widths, as measured between $\approx 100-500 \mathrm{eV}$, do not depend strongly on energy. Thus, we do not expect a large width variation in our results to stem from the cooling of the strahl with radial distance. More importantly, the main results of this study are derived through comparison between sunward and outward distributions in equivalent distance bins. As such, the two will have experienced equivalent cooling when observed in each bin, and so any differences found should be a result of other effects.

\subsection{Outward Strahl}

The results on the evolution of outward directed strahl properties with heliocentric distance provide validation of the methodology of this study. We compare our results of strahl width, $w$, to those of Hammond et al. (1996) and Berčič et al. (2019) in Table 2 for the innermost and outermost distance bins. Both Hammond et al. (1996) and Berčič et al. (2019) present their results as full width at half maximum (FWHM), and so we convert to HWHM with a factor of 1/2. The Hammond et al. (1996) strahl width observations are made with Ulysses 
between $1.3-3.8 \mathrm{AU}$, at $225 \mathrm{eV}$, and they are for fast wind only. We extrapolate their results to the outer bin of the present study $(0.91 \mathrm{AU})$ using the coefficients of a linear fit to those widths, which are listed in their article. While Hammond et al. (1996) use a comparable fitting method to the present study, Berčič et al. (2019) employ a different method, in which the strahl is first isolated by subtracting core and halo components which are determined through fitting the full VDF. The isolated strahl distribution is then fit with a Gaussian function, with no constant background. We expect that $w$ derived from this technique will tend to be wider than is derived here, because the subtraction of a fitted core and halo may allow some strahl signal to remain at higher pitch angles, which our approach would consider to be part of the background. They also bin data by core parallel electron plasma beta, $\beta_{e c \|}$, instead of velocity, and into narrower distance bins. Table 2 confirms our expectation that we derive narrower strahl than Berčič et al. (2019), in comparable distance bins. For the fast wind in the outermost distance bin, we measure very similar median strahl width to Hammond et al. (1996), who employ a similar technique to this study. This comparison thus serves as validation of our methodology and data set.

As introduced in Section 1, we frame results of strahl evolution as a competition between adiabatic focussing and pitch angle scattering, which may lead to increasing strahl width if scattering dominates. Pitch angle scattering may also scatter electrons to pitch angles $>90^{\circ}$, removing electrons from the strahl portion of the distribution entirely. We shall refer to the former type of scattering as "broadening", and the latter as "attenuation". This nomenclature is designed not to comment or speculate upon the physical processes responsible for the scattering. We expect broadening to increase $w$, and attenuation to manifest as a decrease in $A$ (or, more accurately, $\sigma A$, Section 2.2) and increase in $C$.

For outward strahl, we observe both an increase in $w$, and decrease in $\sigma A$ with heliocentric distance, for both fast and slow solar wind. We also observe an increase in $C$ for the fast wind only. We note that $\log (A+C)$ also decreases very similarly to $\log (A)$ for the fast wind, and so we are confident that this increasing $C$ is not the driver of the decrease in amplitude (i.e. it is not the case that the halo component becomes stronger and makes the apparent strahl amplitude weaker). These results suggest that both broadening and attenuation may take place at a sufficient rate to overcome adiabatic focussing effects, over the distance range of $0.3-1 \mathrm{AU}$. These results are also consistent with our expectations based on broadening taking place at greater distances (Hammond et al., 1996; Graham et al., 2017), and also with the observations of broadening (Berčič et al., 2019) and attenuation (Maksimovic et al., 2005; Štverák et al., 2009) observed with the same Helios data set.

The fact that we do not observe an increase in the halo density for the slow wind is the only observation which is not fully consistent with the above studies. $\sigma A$ decreases in the slow wind, suggesting that electrons are scattered, but perhaps in energy as well as pitch angle. This could be explained by the scattering in the slow wind not conserving the energy of the scattered electrons. This explanation is then consistent with the result found by Štverák et al. (2009) that attenuation occurs both in fast and slow wind, as these authors considered the entire halo and strahl components across a range of energies.

\subsection{Sunward Strahl}

We shall now discuss the results for sunward strahl properties, in comparison to those for outward strahl. We interpret these results under the framework for sunward strahl and inverted HMF illustrated in Figure 9. This schematic contrasts the path of propagation for strahl electrons along an inverted $(I)$ and an uninverted and outward $(O)$ field line. We consider the case where identical strahl electron distributions are released at the base of each 
Figure 9 Schematic of heliospheric magnetic field for one field line which is directed outward along its length $(\mathrm{O})$ and one which inverts (I). A pair of heliospheric distances are labelled $R_{a}$ and $R_{b}$. The directions of strahl electron propagation along the field lines are indicated by red arrows, at numbered locations.

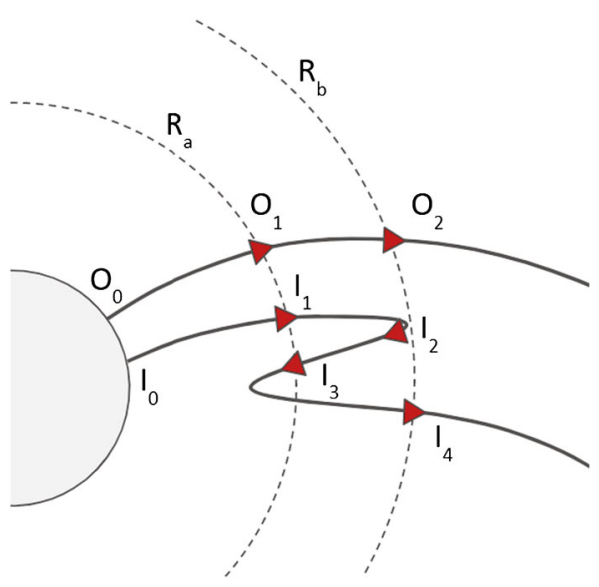

field line, and where the magnetic field strengths along field lines $I$ and $O$ are identical prior to the inversion of $I$. At distance $\mathrm{R}_{\mathrm{a}}$, strahl at points $O_{1}$ and $I_{1}$ have experienced the same degree of focussing, and so the strahl at these two locations are of identical width. Along field line $I$, the strahl electrons travel along the kinked magnetic field, back to $R_{a}$ at point $I_{3}$. Ignoring all scattering effects, we expect the PA width of the strahl here to be comparable to the width at points $O_{1}$ and $I_{1}$, since the focussing/defocussing effects are adiabatic. Therefore, disregarding other variability of the strahl electrons, we can interpret differences between outward and sunward strahl populations, measured at comparable distances, as being a result of non-adiabatic scattering effects which apply because of increased path length. Figure 9 also shows that we might measure sunward strahl at any point along the inverted portion of the field line. Thus, at a given heliocentric distance, the additional path length which applies for sunward strahl is variable.

From points $\mathrm{O}_{2}$ and $I_{4}$ at $\mathrm{R}_{\mathrm{b}}$, Figure 9 also demonstrates that we may measure strahl along an inverted flux tube, but downstream of the inversion. Strahl at $I_{4}$ will have undergone any processing which applies due to the inversion, while strahl at $\mathrm{O}_{2}$ will not. However, the strahl local to both $\mathrm{O}_{2}$ and $\mathrm{I}_{4}$ will be outward directed, and so the two cases are not separable from observations. We do not expect configurations such as $I_{4}$ to have a strong impact on the distribution of parameters for outward strahl in this study, however, because of the relatively low occurrence rate of inverted flux.

Sunward strahl drops off in $\sigma A$, although somewhat less sharply than outward strahl, suggesting that both are subject to degrees of attenuation through scattering. Further, in all distance bins, sunward strahl intensity and $\sigma A$ are lower than the equivalent for outward strahl. We suggest that at each distance bin, sunward strahl is subject to additional scattering in comparison to outward strahl. A simple constant-rate scattering model (e.g. Owens, Crooker, and Schwadron, 2008) can explain this in conjunction with additional path length for sunward strahl (Figure 9). The strahl electrons travel further along the field and are subject to additional scattering. While in the case modelled by Owens, Crooker, and Schwadron (2008) this scattering resulted in broadening of the strahl, this result demonstrates the scattering of electrons out of the strahl population entirely. It cannot be determined from these results alone whether the further scattering of these electrons occurs during only the initial outward portion of their journey ( $I_{1}$ to $\left.I_{2}\right)$, during the additional sunward portion $\left(I_{2}\right.$ to $\left.I_{3}\right)$, or both.

The sunward and outward trend lines for $\log A$ and $\sigma A$ tend to converge in Figures 7 and 8 , particularly for the slow wind. This suggests that the relative importance of the ad- 
ditional scattering on sunward strahl decreases with distance. In terms of a constant-rate scattering model, this may be because, for kinks which exist at these greater distances, the average additional path length caused by the kink in the field is not as large in comparison to the total path length. This is related to the evolution of inverted flux topologies with distance, which is yet unknown. There may also be some contribution from a decreasing scattering rate with distance.

We now consider the evolution of sunward strahl pitch angle width. The overall radial trend of pitch angle width is far less pronounced for sunward strahl than outward strahl; the increase in median measurable width between 0.3 and $1 \mathrm{AU}$ for sunward (outward) strahl is around $12^{\circ}\left(15^{\circ}\right)$ for fast wind and $6^{\circ}\left(18^{\circ}\right)$ for slow wind. The median sunward strahl is notably wider than outward strahl in the two innermost distance bins. We note that the fPADs in Figure 5 suggest that this is true at all distances, however, the appearance of those plots is primarily a result of the bias of sunward strahl occurrence towards the outer distance bins found in Table 1, where the strahl is widest. As sunward strahl is wider than outward strahl nearer the Sun, it appears that the additional path length results also in increased broadening, as we found for attenuation in the above case. As the sunward and outward lines for $A$ and $\sigma A$ were found to tend towards convergence in this distance range, the sunward strahl PA widths too have comparable widths to outward strahl by $\approx 0.9 \mathrm{AU}$. If we interpret this convergence as we did for attenuation, then this result suggests that the additional broadening of the sunward strahl electrons is negligible for these outer distance bins. Again, we can explain this as the additional path length for sunward strahl in these bins being insignificant in comparison to the total path length, leading to less scattering. Alternatively, the rate of broadening may drop off at $\approx 1 \mathrm{AU}$. Convergence occurs closer to the Sun for $w$ than for $A$, which suggests that there may be a different path-length dependence for that scattering which broadens and that which attenuates the strahl.

We find that in the inner two distance bins, the background halo, represented by $C$, is stronger for sunward strahl than for outward strahl PADs in comparable bins. This is complementary to the idea that sunward strahl undergoes more attenuation than outward strahl, as electrons could reasonably be scattered from the strahl into the halo at comparable energies. Sunward and outward strahl also tend to be associated with converging values of $C$ at increasing distances. Again, we interpret this as the relative importance of scattering along the kinked portion of the field line dropping off with increasing heliospheric distance. In Figure 7, we find that the evolution of $C$ for the sunward strahl is similar to the evolution of $C$ for the outward strahl in equivalent streams, decreasing in slow wind and increasing for fast. This is again broadly consistent with the same scattering mechanisms applying for sunward and outward strahl.

In order to quantitatively estimate the additional path lengths necessary to produce the observations for sunward strahl here, modelling is required. This could be relatively simple, perhaps as some extension to the constant-rate scattering model used by Owens, Crooker, and Schwadron (2008). However, to fully reproduce our observations, it may prove necessary to relax this constant scattering rate condition, in favour of a scattering rate with a dependence on heliocentric distance or other parameters. For now, we leave this modelling to future work, as the qualitative approach here appears to be a sufficient first explanation.

Distance-dependent scattering may provide an alternative or complementary explanation for these results. DeForest et al. (2016) find evidence in imaging data for the onset of solar wind turbulence at distances of $44-88 \mathrm{Rs}(\approx 0.2-0.4 \mathrm{AU})$. Here, waves and fluctuations produced by the turbulent cascade (e.g. whistler-mode waves by Saito and Gary, 2007) would first become available to interact with strahl electrons. This may lead to the onset of the strahl scattering inferred from heliospheric observations available thus far. The portion 
of strahl propagation below heliocentric distances $\approx 0.2-0.4$ AU would then presumably lead to weaker scattering than the portion at distances $>0.4 \mathrm{AU}$, when the turbulent cascade is more developed. In the inner distance bins, we would expect a large fraction of the path length for the outward strahl to involve scattering at this lesser rate. However, travel over the additional path length applied to the sunward strahl may subject the electrons to scattering at the higher ( $>0.4 \mathrm{AU})$ rate, and thus sunward strahl should be more scattered than in a constant-rate case (this depends on the scale of the inversion). Conversely, at greater heliospheric distances $(e . g . \approx 1 \mathrm{AU})$ both the outward and sunward strahl should be subject to a more comparable degree of scattering, as the weak-scattering portion of propagation within $\approx 0.2-0.4 \mathrm{AU}$ is less important. This is generally consistent with what we have reported in this study, and so this approximate location of onset for plasma turbulence appears compatible with our results and previous explanation. Confirmation of this argument depends on more detailed modelling of scattering rates, and more accurate knowledge of the size and scales of HMF inversions, and how these evolve with radial distance.

\subsection{Prospects for Future Observations}

We found in the previous section that at distances $\approx 1$ AU the properties of sunward and outward strahl are most similar. Thus, at time of writing, these statistical results could only have been obtained through analysis of the Helios data set. Further study of this evolution of sunward strahl electrons will therefore only be possible in the future with the recently-launched Parker Solar Probe (PSP: Fox et al., 2016) and upcoming Solar Orbiter (SO: Müller et al., 2013) missions. The electron instruments on both spacecraft (the PSP/Solar Wind Electrons Alphas and Protons experiment, and the SO/Electron Analyzer System; Kasper et al., 2016; Müller et al., 2013) will perform at a level far superior to Helios I2, and so many of the caveats and limitations which were necessary for the data in this article will not be necessary. For example, the greater angular resolution of both instruments will allow for characterisation of strahl with PA width $<15^{\circ}$. This will remove a major speculative aspect from this study regarding the piled-up samples at around $15^{\circ}$. Further, observations of the strahl when the HMF lies out of the ecliptic plane will allow study of inner-heliosphere strahl during cases (flux ropes, SIRs, periods of strong fluctuation) neglected here. PSP in particular will observe the strahl at distances very close to its formation, such that its evolution can be measured across a broad range of the inner heliosphere.

\section{Conclusions}

Subject to the caveats laid out in the previous section, in this study we have quantified the properties of sunward and outward strahl electrons using the Helios 1 data set. For the first time, we have statistically compared the properties and evolution of sunward strahl to outward strahl. These results give us new insight into the scattering of strahl electrons and the evolution of inverted flux in the heliosphere.

We find that sunward strahl is typically broader, has lower amplitude, and is associated with a stronger halo component than normal outward strahl. These findings suggest that sunward strahl is subject to increased scattering which outward strahl is not. We attribute this increased scattering to additional propagation along the magnetic field. This is consistent with the prevailing view of sunward strahl, i.e. that it arises due to strahl flowing along locally inverted field lines. Increased scattering is broadly compatible with both a constantrate scattering model for the strahl (Owens, Crooker, and Schwadron, 2008), as well as 
scattering which primarily initiates with the onset of solar wind turbulence in the inner heliosphere (e.g. DeForest et al., 2016).

A more quantitative explanation of the above results will require modelling of the candidate scattering processes for strahl propagating along different magnetic topologies, which we intend to pursue as future work. Additional follow-on work will also be centred around the expected improvements to observations and coverage (in heliocentric distance as well as azimuthal angle) of PSP and SO. This study thus opens avenues for future research which will build knowledge of the evolution, and ultimately the origins, of solar wind strahl electrons and inverted heliospheric flux.

Acknowledgements This work was part-funded by the Science and Technology Facilities Council (STFC) grant No. ST/M000885/1 and ST/R000921/1, and the Natural Environment Research Council (NERC) grant No. NE/P016928/1. CJO is supported by STFC grant No. ST/S000240/1. We acknowledge all members of the Helios data archive team ${ }^{1}$ who made the Helios data publicly available to the space physics community. We thank David Stansby for making available the new Helios proton core data set. ${ }^{2}$ This research made use of Astropy, ${ }^{3}$ a community-developed core Python package for Astronomy (Robitaille and Astropy Collaboration, 2013; Astropy Collaboration, 2018). This research made use of HelioPy, a community-developed Python package for space physics (Stansby et al., 2019). Figures were produced using the Matplotlib plotting library for Python (Hunter, 2007). This work was discussed at the ESA Solar Wind Electron Workshop which was supported by the Faculty of the European Space Astronomy Centre (ESAC). The authors thank Laura Berčič, Georgina Graham, Davin Larson, and Georgios Nicolau for useful discussions.

Disclosure of Potential Conflicts of Interest The authors declare that there are no conflicts of interest.

Publisher's Note Springer Nature remains neutral with regard to jurisdictional claims in published maps and institutional affiliations.

Open Access This article is licensed under a Creative Commons Attribution 4.0 International License, which permits use, sharing, adaptation, distribution and reproduction in any medium or format, as long as you give appropriate credit to the original author(s) and the source, provide a link to the Creative Commons licence, and indicate if changes were made. The images or other third party material in this article are included in the article's Creative Commons licence, unless indicated otherwise in a credit line to the material. If material is not included in the article's Creative Commons licence and your intended use is not permitted by statutory regulation or exceeds the permitted use, you will need to obtain permission directly from the copyright holder. To view a copy of this licence, visit http://creativecommons.org/licenses/by/4.0/.

\section{Appendix A: Bulk Flow Corrections}

Here we demonstrate the importance of the spacecraft-solar wind frame correction applied in Section 2 for electrons at suprathermal energies. To model the importance of solar wind flow on suprathermal electron distributions, we first construct model suprathermal distributions using a kappa function, which has been found to be appropriate for suprathermal electron populations by Maksimovic et al. (2005) and Štverák et al. (2009). The function is defined as (Baumjohann and Treumann, 1997):

$$
f(E)=n\left(\frac{m}{2 \pi \kappa E_{0}}\right)^{3 / 2} \frac{\Gamma(\kappa+1)}{\Gamma(\kappa-1 / 2)}\left(1+\frac{E^{*}}{\kappa E_{0}}\right)^{-(\kappa+1)},
$$

\footnotetext{
${ }^{1}$ http://helios-data.ssl.berkeley.edu/team-members/.

${ }^{2} \mathrm{DOI}$.

${ }^{3}$ http://www.astropy.org.
} 
Figure 10 Plot of

$\left(f_{+}-f_{-}\right) / f_{0}$ against energy,

where $f_{0}$ is a kappa distribution with $T=50 \mathrm{eV}$, and arbitrary $n$, centred around $E=0$. The orange line is for $\kappa=2.5$ and the blue line for $\kappa \rightarrow \infty$. $f_{+}\left(f_{-}\right)$ represents $f_{0}$ when shifted by a bulk flow velocity of $500 \mathrm{~km} \mathrm{~s}^{-1}$, sampled in the anti-sunward (sunward) direction in the spacecraft frame.

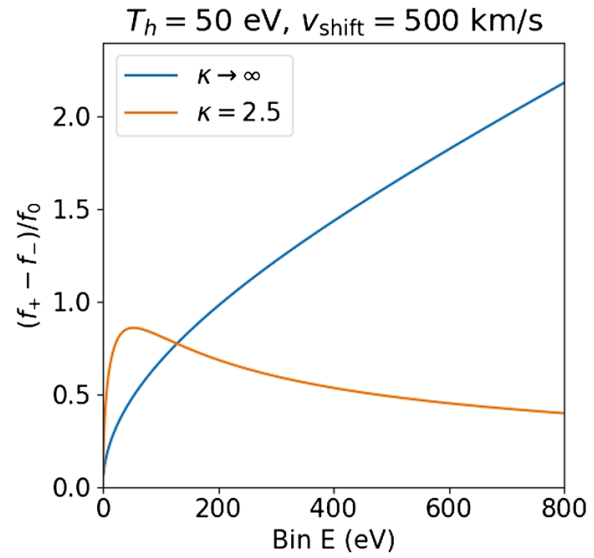

where $\Gamma$ represents the gamma function, $\kappa$ is a dimensionless parameter $>1.5, E$ is particle energy, $E_{0}=k_{B} T_{s}(1-3 / 2 \kappa)$ is the mean particle energy $\left(k_{B}\right.$ is the Boltzmann constant and $T_{s}$ is the suprathermal temperature), and $E^{*}=\left(\sqrt{E}-\sqrt{E_{s}}\right)^{2}$, for a shift energy $E_{s}$. We obtain suitable values for $n, E_{0}$, and $\kappa$ from studies of solar wind suprathermal electrons at $1 \mathrm{AU}$ (e.g. Tao et al., 2016; Macneil, Owen, and Wicks, 2017), and shift $E^{*}$ based on the bulk flow. We do not include a core electron component, as we expect the core to make negligible contribution to the VDF at suprathermal energies.

A single-energy cut through a distribution $f_{0}$, which is isotropic in the solar wind frame, will appear anisotropic in the spacecraft frame. The largest deviation from $f_{0}$ will manifest in the samples in the anti-sunward and sunward directions in the spacecraft frame, $f_{+}$ and $f_{-} . f_{+}$and $f_{-}$can be calculated by shifting Equation 3 upwards and downwards by the bulk speed respectively. $\left(f_{+}-f_{-}\right) / f_{0}$ then quantifies the variation seen when viewing a PAD, which is flat in the solar wind frame, in the spacecraft frame. Figure 10 shows $\left(f_{+}-f_{-}\right) / f_{0}$ as a function of bin energy $E$, for $\kappa$ values of 2.5 and $\rightarrow \infty$ (the Maxwellian limit), halo temperature $T_{s}=50 \mathrm{eV}$, and bulk speed of $500 \mathrm{~km} \mathrm{~s}^{-1} .\left(f_{+}-f_{-}\right) / f_{0}$ in the Maxwellian case increases indefinitely with bin energy, while in the $\kappa=2.5$ case tapers off following a maximum at $\approx 40 \mathrm{eV}$. For energies at which we measure the strahl population in this study $(\gtrsim 200 \mathrm{eV}),\left(f_{+}-f_{-}\right) / f_{0}$ ranges from $\approx 0.5-2$. This size of variation is sufficient to affect the properties of the combined halo and strahl populations, viewed as PADs in Section 3, and affect the classification of strahl in Section 2.1. The core and halo electrons make up the background component of the PADs analysed in this study, which we represent with the constant $C$. The shift of electron VDFs from the spacecraft to the bulk solar wind frame, in which the core and halo populations will appear more isotropic, thus improves the characterisation of the strahl population.

The process of correcting electron VDFs into the bulk solar wind frame is described in Section 2. Figure 11 shows normalised cuts at a range of energies through an example VDF measured by Helios 1, as a function of look direction in the uncorrected spacecraft frame (left), and corrected into the solar wind frame (right). The low-energy electrons are far closer to isotropy in the solar wind frame than in the spacecraft frame, no longer peaking strongly around $0^{\circ}$. The suprathermal $(E \gtrsim 80 \mathrm{eV})$ electrons exhibit a strahl, which here peaks at look angle $\approx-100^{\circ}$. Following the correction to the solar wind frame, these peaks no longer skew strongly towards the bulk flow direction $\left(0^{\circ}\right)$; the strahl appears gyrotropic. The correction also has a variable effect on the relative peak value of the strahl at different energies. 

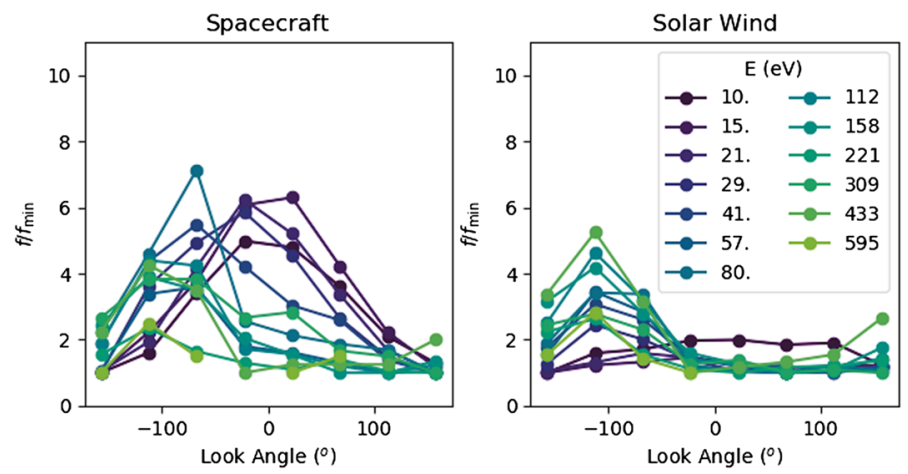

Figure 11 Left: Plot of phase space density against look direction at a range of energies for the same example VDF measured by Helios 1. The values are normalised by the minimum at each energy bin. Right: The same plot for the same example VDF, at equivalent energy bins, but now corrected to the solar wind frame as described in Section 2.
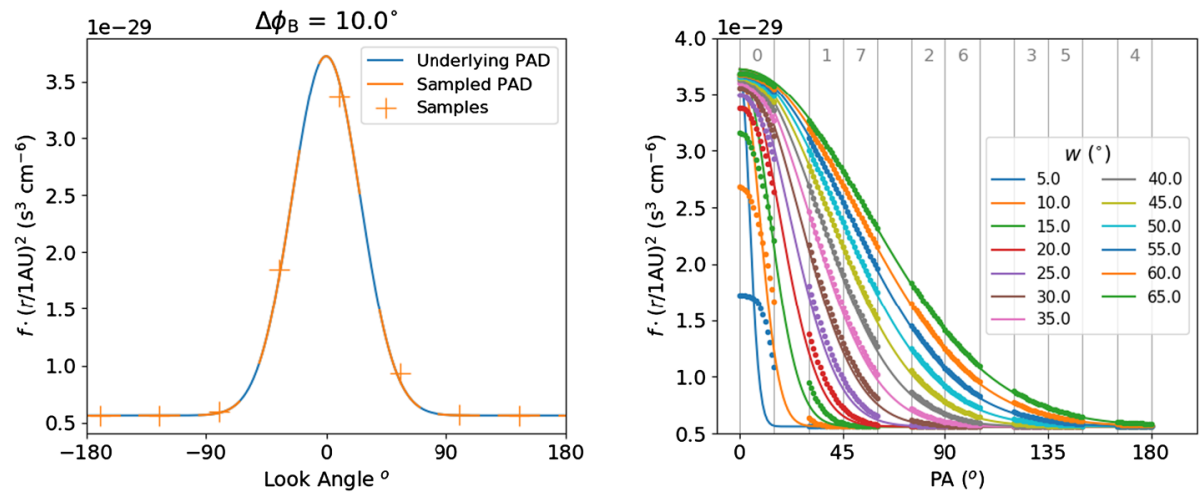

Figure 12 Left: Example simulated PA distribution at a single energy, used to simulate the Helios observations (solid line). The distribution is plotted as a function of pitch angle, but it is mirrored at negative pitch angles as this enables better illustration of the sampling process. Also shown are sampled values of the distribution (orange crosses) at eight PAs. The line is highlighted in orange to correspond to portions of the underlying distribution which contribute to each sample. Right: Solid coloured lines show several simulated PA distributions with the same value of $A$ and $C$, but varying $w$. Dots show sampled values of each distribution, as described in the text, at selected pitch angles, with colours indicating the corresponding underlying distribution. Angles spanned by the eight PA bins of the instrument, which are numbered as in Figure 1b, are separated by vertical lines. For each PA bin, the different samples correspond to different values of $\Delta \phi_{\mathrm{B}}$ in the range $\left[0^{\circ}, 15^{\circ}\right]$, in $1^{\circ}$ intervals.

The model of Figure 10 and example of Figure 11 demonstrate that the shift to the bulk solar wind frame makes the suprathermal PADs more physically representative, and it has a non-negligible impact on the observed properties of the suprathermal PADs. Leaving the data uncorrected affects the observed width and amplitude of the strahl. We note that the bulk flow will obscure the signal from sunward strahl populations in particular, as they propagate partially against the solar wind flow direction. This correction is thus particularly important for the present study to maximise the number of sunward strahl distributions which can be identified. 

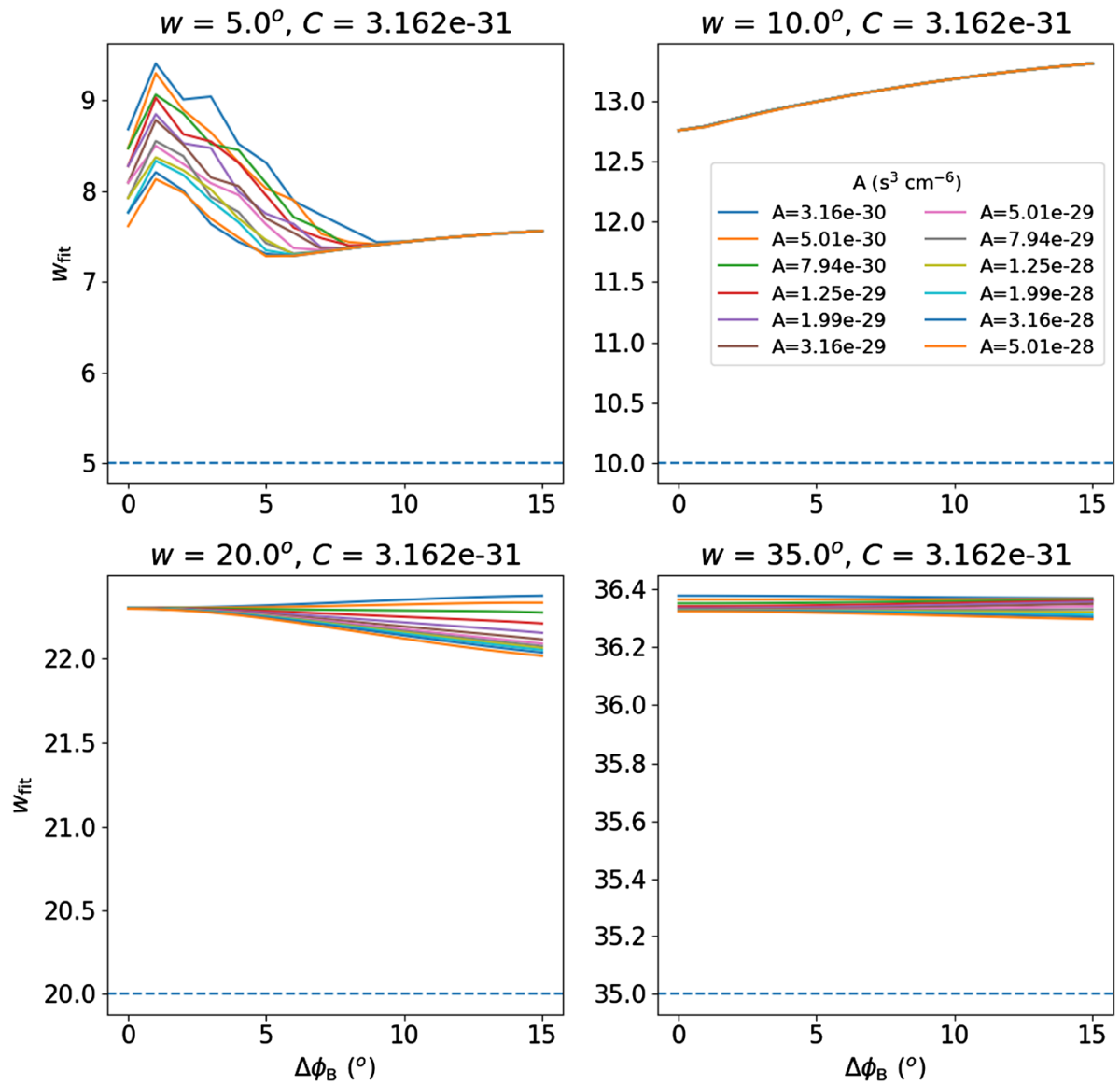

Figure 13 Fitted strahl width, $w_{\text {fit }}$, derived from fitting underlying distributions of varying amplitude $A$, plotted for distributions sampled at different $\Delta \phi_{B}$. Each panel corresponds to an underlying distribution of different true width, $w$, indicated by the blue dashed line.

\section{Appendix B: Evaluation of the Fitting Procedure}

The incomplete angular coverage and large bin width of Helios 1 may limit our ability to derive the true properties of measured PADs. In this appendix we aim to understand these limitations more quantitatively. We first simulate the sampling of an underlying electron distribution by the Helios plasma instrument. We model slices of 2-dimensional PA distributions at a single energy, using a Gaussian distribution function (Equation 1) in the range $\left[-180^{\circ}, 180^{\circ}\right]$. We use ranges of the three parameters $w, A$, and $C$, which we choose based on examination of distributions measured by Helios 1. The left panel of Figure 12 shows an example of such an underlying distribution as a solid blue line of $f$ against look angle. We sample these distributions about the central angles of the eight PA bins using the angular coverage shown for Helios 1 in Figure $1 \mathrm{~b}$, producing the sampled distribution $f_{s}$. We assume that the magnetic field lies perfectly in the ecliptic plane, such that $f_{s}$ captures the strahl. Each sample is a mean of a portion of the underlying distribution spanning $\pm 15^{\circ}$ (the azimuthal bin width $d \theta$ ) about the bin angle. The blue line in the left panel of Figure 12 is 

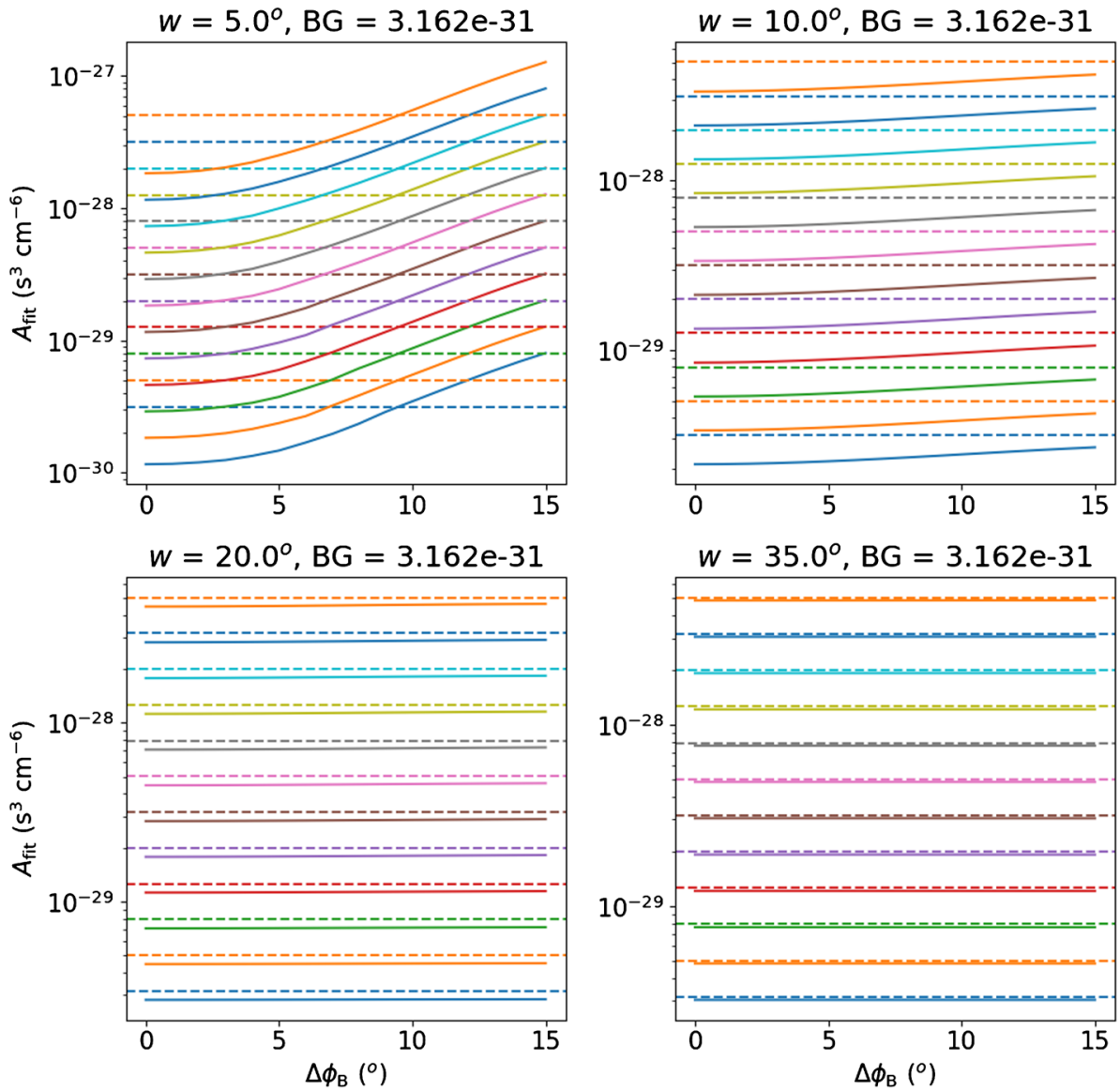

Figure 14 Fitted strahl amplitude, $A_{\text {fit }}$ (solid lines), derived from fitting underlying distributions of varying amplitude $A$ (dashed lines of the same colour), plotted for distributions sampled at different $\Delta \phi_{B}$. Each panel corresponds to an underlying distribution of different true width $w$.

highlighted in orange to show portions of the underlying distribution which contributed to each sample, given $\Delta \phi_{\mathrm{B}}=10^{\circ}$. The sampled mean values are shown as orange crosses.

The right panel of Figure 12 shows mean sampled values in the eight angular bins, for a range of underlying distributions of $w=\left[5^{\circ}, 65^{\circ}\right]$, at fixed $A$ and $C$. The sampled values are shown for a range of angles of $\Delta \phi_{\mathrm{B}}=\left[0^{\circ}, 15^{\circ}\right]$ as coloured dots, and corresponding underlying distributions are shown in the same colour as solid lines. A single sampled distribution $f_{s}$ will be made up of eight samples, with one drawn from each PA bin, at angles dependent on $\Delta \phi_{\mathrm{B}}$. From this plot, we see that $\Delta \phi_{\mathrm{B}}$ can strongly affect the values of $f_{s}$, particularly in the PA bin nearest $0^{\circ}, f_{s 0}$. For example, for $\Delta \phi_{\mathrm{B}}$ approaching $0^{\circ}$ (meaning sample $f_{s 0}$ is made very close to $\left.0^{\circ}\right) f_{s 0}$ underestimates the true value of $f$. The inaccuracy of this sample is worst for the narrowest distributions of $w \leq 10^{\circ}$. We note further that for the distributions of $w \leq 10^{\circ}$, only samples in bin 0 contain some signal greater than the background value, $C$. In other words, only this bin samples any strahl electrons, and the other bins sample halo only. 
Next, we apply the same Gaussian fitting procedure used in Section 2.2 to the simulated sampled distributions $f_{s}$. As an evaluation of the fitting method, we compare fitted parameters $w_{\text {fit }}, A_{\text {fit }}$, and $C_{\text {fit }}$ with the true parameters of the original distributions, $w, A$, and $C$. In all cases, we find that $C_{\text {fit }}$ is very close to $C$. Thus, moving forward we only evaluate $w_{\text {fit }}$ and $A_{\text {fit }}$. Figure 13 shows four plots, each of $w_{\text {fit }}$ against $\Delta \phi_{\mathrm{B}}$ for distributions with a range of $A$ and fixed $C$. The plots are for distributions with underlying values of $w=5^{\circ}, 10^{\circ}, 20^{\circ}$, and $35^{\circ}$, shown as blue dashed lines. In all cases $w_{\text {fit }}$ is an overestimate, with the narrower distributions being farther from $w$. The narrowest cases can overestimate by several degrees. The dependence of $w_{\text {fit }}$ on $\Delta \phi_{\mathrm{B}}$ and $A$ is different for each case.

Figure 14 has a similar format to Figure 13, now comparing values of $A_{\text {fit }}$ (solid lines) to $A$ (dashed lines of the same colour) for underlying distributions of $w=5^{\circ}, 10^{\circ}, 20^{\circ}$, and $35^{\circ}$. Again the fitting procedure performs better for distributions of greater $w$. Distributions of $w \geq 10^{\circ}$ are underestimated in $A_{\text {fit }}$, with distributions sampled at higher $\Delta \phi_{\mathrm{B}}$ being slightly more accurate. The $w=5^{\circ}$ distributions are underestimated in $A_{\text {fit }}$ significantly for $\Delta \phi_{\mathrm{B}} \lesssim 10^{\circ}$, and overestimated for $\Delta \phi_{\mathrm{B}} \gtrsim 10^{\circ}$.

We recall that for distributions of $w \lesssim 10^{\circ}$, the strahl is only sampled in bin 0 . Fitting such distributions, both $A_{\text {fit }}$ and $w_{\text {fit }}$ are fitted from only a single data point which lies above the background. The fitting procedure for such distributions is thus under-constrained for the strahl component only. The $w_{\text {fit }}$ and $A_{\text {fit }}$ results in Figures 13 and 14 for $w \lesssim 10^{\circ}$ thus likely represent only the biases of the fitting algorithm itself.

We arrive at the conclusion that our fitted results of $w_{\text {fit }}$ and $A_{\text {fit }}$ for the Helios 1 data, for distributions of underlying width $w \lesssim 10^{\circ}$, are unreliable. Based on Figure 13, the fitting procedure is likely to overestimate such widths, and might under or overestimate $A$. We also expect the fitting errors for such distributions to often be large, and so many should be rejected based on our error tolerance. Since $w$ for these narrow distributions is overestimated by $3-5^{\circ}$, we do not trust distributions with fitted widths of $w_{\text {fit }} \lesssim 15^{\circ}$, and expect that the true underlying distribution widths may be considerably narrower.

\section{References}

Anderson, B., Skoug, R., Steinberg, J., McComas, D.: 2012, Variability of the solar wind suprathermal electron strahl. J. Geophys. Res. Space Phys. 117(A4), A04107. DOI.

Antiochos, S., DeVore, C., Karpen, J., Mikić, Z.: 2007, Structure and dynamics of the Sun's open magnetic field. Astrophys. J. 671(1), 936. DOI.

Astropy Collaboration: 2018, The astropy project: Building an open-science project and status of the v2.0 core package. Astron. J. 156, 123. DOI.

Baker, D., Rouillard, A.P., van Driel-Gesztelyi, L., Démoulin, P., Harra, L.K., Lavraud, B., Davies, J.A., Opitz, A., Luhmann, J.G., Sauvaud, J.-A., Galvin, A.B.: 2009, Signatures of interchange reconnection: STEREO, ACE and Hinode observations combined. Ann. Geophys. 27, 3883. DOI. ADS.

Baumjohann, W., Treumann, R.: 1997, Basic Space Plasma Physics, World Scientific, River Edge. DOI.

Berčič, L., Maksimović, M., Landi, S., Matteini, L.: 2019, Scattering of strahl electrons in the solar wind between 0.3 and 1 AU: Helios observations. Mon. Not. Roy. Astron. Soc. DOI.

Che, H., Goldstein, M.: 2014, The origin of non-Maxwellian solar wind electron velocity distribution function: Connection to nanoflares in the solar corona. Astrophys. J. 795(2), L38. DOI.

Crooker, N., Gosling, J., Kahler, S.: 2002, Reducing heliospheric magnetic flux from coronal mass ejections without disconnection. J. Geophys. Res. 107(A2), 1028. DOI.

Crooker, N., Owens, M.: 2012, Interchange reconnection: Remote sensing of solar signature and role in heliospheric magnetic flux budget. Space Sci. Rev. 172(1-4), 201. DOI.

Crooker, N., Pagel, C.: 2008, Residual strahls in solar wind electron dropouts: Signatures of magnetic connection to the Sun, disconnection, or interchange reconnection? J. Geophys. Res. 113(A2), A02106. DOI. 
Crooker, N., Kahler, S., Larson, D., Lin, R.: 2004, Large-scale magnetic field inversions at sector boundaries. J. Geophys. Res. 109(A3), A03108. DOI.

DeForest, C., Matthaeus, W., Viall, N., Cranmer, S.: 2016, Fading coronal structure and the onset of turbulence in the young solar wind. Astrophys. J. 828(2), 66. DOI.

Feldman, W., Asbridge, J., Bame, S., Montgomery, M.D., Gary, S.P.: 1975, Solar wind electrons. J. Geophys. Res. 80(31), 4181. DOI.

Feldman, W., Asbridge, J., Bame, S., Gosling, J., Lemons, D.: 1978, Electron heating within interaction zones of simple high-speed solar wind streams. J. Geophys. Res. 83(A11), 5297. DOI.

Fisk, L.: 2003, Acceleration of the solar wind as a result of the reconnection of open magnetic flux with coronal loops. J. Geophys. Res. Space Phys. 108(A4), 1157. DOI.

Fisk, L., Schwadron, N., Zurbuchen, T.: 1998, On the slow solar wind. Space Sci. Rev. 86(1-4), 51. DOI.

Fox, N., Velli, M., Bale, S., Decker, R., Driesman, A., Howard, R., Kasper, J., Kinnison, J., Kusterer, M., Lario, D., et al.: 2016, The solar probe plus mission: Humanity's first visit to our star. Space Sci. Rev. 204(1-4), 7. DOI.

Gosling, J., Baker, D., Bame, S., Feldman, W., Zwickl, R., Smith, E.: 1987, Bidirectional solar wind electron heat flux events. J. Geophys. Res. 92(A8), 8519. DOI.

Gosling, J., Skoug, R., McComas, D., Smith, C.: 2005, Direct evidence for magnetic reconnection in the solar wind near 1 AU. J. Geophys. Res. 110(A1), A01107. DOI.

Graham, G., Rae, I., Owen, C., Walsh, A., Arridge, C., Gilbert, L., Lewis, G., Jones, G., Forsyth, C., Coates, A., et al.: 2017, The evolution of solar wind strahl with heliospheric distance. J. Geophys. Res. 122(4), 3858. DOI.

Gurnett, D.A., Anderson, R.R.: 1977, Plasma wave electric fields in the solar wind: Initial results from Helios 1. J. Geophys. Res. 82(4), 632. DOI.

Hammond, C., Feldman, W., McComas, D., Phillips, J., Forsyth, R.: 1996, Variation of electron-strahl width in the high-speed solar wind: Ulysses observations. Astron. Astrophys. 316, 350.

Heidrich-Meisner, V., Peleikis, T., Kruse, M., Berger, L., Wimmer-Schweingruber, R.: 2016, Observations of high and low Fe charge states in individual solar wind streams with coronal-hole origin. Astron. Astrophys. 593, A70. DOI.

Hellinger, P., Trávníček, P.M., Decyk, V.K., Schriver, D.: 2014, Oblique electron fire hose instability: Particlein-cell simulations. J. Geophys. Res. 119(1), 59. DOI.

Horaites, K., Boldyrev, S., Medvedev, M.V.: 2018, Electron strahl and halo formation in the solar wind. Mon. Not. Roy. Astron. Soc. 484(2), 2474. DOI.

Hunter, J.D.: 2007, Matplotlib: A 2D graphics environment. Comput. Sci. Eng. 9(3), 90. DOI.

Kahler, S., Lin, R.: 1994, The determination of interplanetary magnetic field polarities around sector boundaries using $E>2 \mathrm{keV}$ electrons. Geophys. Res. Lett. 21(15), 1575. DOI.

Kajdič, P., Alexandrova, O., Maksimovic, M., Lacombe, C., Fazakerley, A.: 2016, Suprathermal electron strahl widths in the presence of narrow-band whistler waves in the solar wind. Astrophys. J. 833(2), 172. DOI.

Kasper, J.C., Abiad, R., Austin, G., Balat-Pichelin, M., Bale, S.D., Belcher, J.W., et al.: 2016, Solar wind electrons alphas and protons (SWEAP) investigation: Design of the solar wind and coronal plasma instrument suite for solar probe plus. Space Sci. Rev. 204(1-4), 131. DOI.

Landi, S., Matteini, L., Pantellini, F.: 2012, On the competition between radial expansion and coulomb collisions in shaping the electron velocity distribution function: Kinetic simulations. Astrophys. J. 760(2), 143. DOI.

Lei, J., Thayer, J.P., Wang, W., McPherron, R.L.: 2011, Impact of cir storms on thermosphere density variability during the solar minimum of 2008. Solar Phys. 274(1-2), 427. DOI.

Lockwood, M., Owens, M.J., Macneil, A.R.: 2019, On the origin of ortho-gardenhose heliospheric flux. Solar Phys. 294(6), 85. DOI.

Lockwood, M., Owens, M., Rouillard, A.: 2009, Excess open solar magnetic flux from satellite data: 2. A survey of kinematic effects. J. Geophys. Res. 114(A11), A11104. DOI.

Macneil, A.R., Owen, C.J., Wicks, R.T.: 2017, Tests for coronal electron temperature signatures in suprathermal electron populations at 1 AU. Ann. Geophys. 35, 1275. DOI.

Maksimovic, M., Zouganelis, I., Chaufray, J.-Y., Issautier, K., Scime, E., Littleton, J., Marsch, E., McComas, D., Salem, C., Lin, R., et al.: 2005, Radial evolution of the electron distribution functions in the fast solar wind between 0.3 and 1.5 AU. J. Geophys. Res. 110(A9), A09104. DOI.

McComas, D., Gosling, J., Phillips, J., Bame, S., Luhmann, J., Smith, E.: 1989, Electron heat flux dropouts in the solar wind: Evidence for interplanetary magnetic field reconnection? J. Geophys. Res. 94(A6), 6907. DOI.

Müller, D., Marsden, R., St. Cyr, O., Gilbert, H.: 2013, Solar Orbiter 285, 25. DOI. 
Owens, M., Crooker, N.: 2007, Reconciling the electron counterstreaming and dropout occurrence rates with the heliospheric flux budget. J. Geophys. Res. 112(A6), A06106. DOI.

Owens, M., Crooker, N., Lockwood, M.: 2013, Solar origin of heliospheric magnetic field inversions: Evidence for coronal loop opening within pseudostreamers. J. Geophys. Res. 118(5), 1868. DOI.

Owens, M., Crooker, N., Schwadron, N.: 2008, Suprathermal electron evolution in a Parker spiral magnetic field. J. Geophys. Res. 113(11), 1.

Owens, M.J., Arge, C., Crooker, N., Schwadron, N., Horbury, T.: 2008, Estimating total heliospheric magnetic flux from single-point in situ measurements. J. Geophys. Res. 113(A12), A12103. DOI.

Owens, M., Lockwood, M., Riley, P., Linker, J.: 2017, Sunward strahl: A method to unambiguously determine open solar flux from in situ spacecraft measurements using suprathermal electron data. J. Geophys. Res. 122(11), 10,980. DOI.

Owens, M.J., Lockwood, M., Barnard, L.A., Macneil, A.R.: 2018, Generation of inverted heliospheric magnetic flux by coronal loop opening and slow solar wind release. Astrophys. J. Lett. 868(1), L14. DOI.

Pagel, C., Crooker, N., Larson, D.: 2005, Assessing electron heat flux dropouts as signatures of magnetic field line disconnection from the Sun. Geophys. Res. Lett. 32(14), L14105. DOI.

Pagel, C., Crooker, N., Larson, D., Kahler, S., Owens, M.: 2005, Understanding electron heat flux signatures in the solar wind. J. Geophys. Res. 110(A1), A01103. DOI.

Pavan, J., Viñas, A., Yoon, P.H., Ziebell, L.F., Gaelzer, R.: 2013, Solar wind strahl broadening by selfgenerated plasma waves. Astrophys. J. Lett. 769(2), L30. DOI.

Phillips, J., Gosling, J., McComas, D., Bame, S., Feldman, W.: 1992, Quantitative analysis of bidirectional electron fluxes within coronal mass ejections at 1 AU. In: Solar Wind Seven Colloquium 1, 651. DOI.

Pierrard, V., Maksimovic, M., Lemaire, J.: 1999, Electron velocity distribution functions from the solar wind to the corona. J. Geophys. Res. 104(A8), 17021. DOI.

Pierrard, V., Maksimovic, M., Lemaire, J.: 2001, Core, halo and strahl electrons in the solar wind. Astrophys. Space Sci. 277(1-2), 195.

Pilipp, W., Muehlhaeuser, K.-H., Miggenrieder, H., Montgomery, M., Rosenbauer, H.: 1987a, Characteristics of electron velocity distribution functions in the solar wind derived from the Helios plasma experiment. J. Geophys. Res. 92(5), 1075. DOI.

Pilipp, W., Miggenrieder, H., Montgomery, M., Mühlhäuser, K.-H., Rosenbauer, H., Schwenn, R.: 1987b, Unusual electron distribution functions in the solar wind derived from the Helios plasma experiment: Double-strahl distributions and distributions with an extremely anisotropic core. J. Geophys. Res. 92(A2), 1093. DOI.

Roberts, A., King, J.: 2008, General information about Helios initial ascii files. https://cdaweb.gsfc.nasa.gov/ misc/NotesH.html\#HELIOS2_40SEC_MAG-PLASMA.

Robitaille, T.P., Astropy Collaboration: 2013, Astropy: A community Python package for astronomy. Astron. Astrophys. 558, A33. DOI.

Rosenbauer, H., Schwenn, R., Marsch, E., Meyer, B., Miggenrieder, H., Montgomery, M., Mühlhäuser, K.H., Pilipp, W., Voges, W., Zink, S.: 1977, A survey on initial results of the Helios plasma experiment. J. Geophys. 42, 561.

Saito, S., Gary, S.: 2007, Whistler scattering of suprathermal electrons in the solar wind: Particle-in-cell simulations. J. Geophys. Res. Space Phys. 112(6), 1. DOI.

Schwadron, N., Fisk, L., Zurbuchen, T.: 1999, Elemental fractionation in the slow solar wind. Astrophys. J. 521(2), 859. DOI.

Schwenn, R., Rosenbauer, H., Miggenrieder, H.: 1975, Das plasmaexperiment auf helios (e1). Raumfahrtforschung 19(5), 226.

Scudder, J., Olbert, S.: 1979, A theory of local and global processes which affect solar wind electrons. II Experimental support. J. Geophys. Res. 84(9), 6603. DOI.

Stansby, D., Salem, C., Matteini, L., Horbury, T.: 2018, A new inner heliosphere proton parameter dataset from the Helios mission. Solar Phys. 293(11), 155. DOI.

Stansby, D., Rai, Y., Broll, J., Shaw, S.: 2019, Aditya: heliopython/heliopy: Heliopy 0.6.7. DOI.

Štverák, Š., Trávníček, P.M., Hellinger, P.: 2015, Electron energetics in the expanding solar wind via Helios observations. J. Geophys. Res. 120(10), 8177. DOI.

Štverák, Š., Maksimovic, M., Travinicek, P., Marsch, E., Fazakerley, A., Scime, E.: 2009, Radial evolution of nonthermal electron populations in the low-latitude solar wind: Helios, Cluster, and Ulysses Observations. J. Geophys. Res. 114(5), 1. DOI.

Tao, J., Wang, L., Zong, Q., Li, G., Salem, C., Wimmer-Schweingruber, R., He, J., Tu, C., Bale, S.: 2016, Quiet-time suprathermal $(\sim 0.1-1.5 \mathrm{keV})$ electrons in the solar wind. Astrophys. J. 820(1), 22. DOI.

Viñas, A., Gurgiolo, C., Nieves-Chinchilla, T., Gary, S., Goldstein, M.: 2010, Whistler waves driven by anisotropic strahl velocity distributions: Cluster observations. In: AIP Conf. Proc. 1216, 265. DOI.

Virtanen, P., Gommers, R., Oliphant, T.E., Haberland, M., Reddy, T., Cournapeau, D., et al.: 2019, SciPy 1.0 - Fundamental Algorithms for Scientific Computing in Python. arXiv e-prints. ADS. 
Vocks, C., Mann, G.: 2003, Generation of suprathermal electrons by resonant wave-particle interaction in the solar corona and wind. Astrophys. J. 593(2), 1134. DOI.

Vocks, C., Salem, C., Lin, R., Mann, G.: 2005, Electron halo and strahl formation in the solar wind by resonant interaction with whistler waves. Astrophys. J. 627(1), 540. DOI. 